\title{
Elafibranor Inhibits Chronic Kidney Disease Progression in NASH Mice
}

\author{
Hung-Cheng Tsai, ${ }^{1,2}$ Fu-Pang Chang, ${ }^{2,3}$ Tzu-Hao Li, ${ }^{2,4,5,6}$ Chih-Wei Liu, ${ }^{2,4,5}$ \\ Chia-Chang Huang, ${ }^{1,5}$ Shiang-Fen Huang, ${ }^{2,7}$ Ying-Ying Yang $\odot{ }^{2,5,8,9}$ Kuei-Chuan Lee, ${ }^{2,8}$ \\ Yun-Cheng Hsieh, ${ }^{2,8}$ Ying-Wen Wang, ${ }^{2,8}$ Tzung-Yan Lee $\mathbb{D}^{2,10}$ Yi-Hsiang Huang $\mathbb{D}^{2,5,8}$ \\ Ming-Chih Hou, ${ }^{2,8}$ and Han-Chieh Lin $\mathbb{D}^{2,8}$ \\ ${ }^{1}$ Department of Medicine, Taipei Veterans General Hospital, Taipei, Taiwan \\ ${ }^{2}$ Department of Medicine, National Yang-Ming University School of Medicine, Taipei, Taiwan \\ ${ }^{3}$ Department of Pathology, Taipei Veterans General Hospital, Taipei, Taiwan \\ ${ }^{4}$ Division of Allergy and Immunology, Taipei Veterans General Hospital, Taipei, Taiwan \\ ${ }^{5}$ Institute of Clinical Medicine, National Yang-Ming University School of Medicine, Taipei, Taiwan \\ ${ }^{6}$ Chia-Yi Branch of Taichung Veterans General Hospital, Chiayi, Taiwan \\ ${ }^{7}$ Division of Infection, Taipei Veterans General Hospital, Taipei, Taiwan \\ ${ }^{8}$ Division of Gastroenterology and Hepatology, Taipei Veterans General Hospital, Taipei, Taiwan \\ ${ }^{9}$ Division of General Medicine, Taipei Veterans General Hospital, Taipei, Taiwan \\ ${ }^{10}$ Graduate Institute of Traditional Chinese Medicine, Chang Guang Menorial Hospital, Linkou, Taiwan
}

Correspondence should be addressed to Ying-Ying Yang; yangyy@vghtpe.gov.tw and Han-Chieh Lin; hclin@vghtpe.gov.tw

Received 16 February 2019; Revised 16 April 2019; Accepted 14 May 2019; Published 19 June 2019

Academic Editor: Koichiro Wada

Copyright (C) 2019 Hung-Cheng Tsai et al. This is an open access article distributed under the Creative Commons Attribution License, which permits unrestricted use, distribution, and reproduction in any medium, provided the original work is properly cited.

Identification of new pharmacological approaches to inhibit the excessive fat intake-induced steatohepatitis and chronic kidney disease $(\mathrm{CKD})$ is important. High-fat diet (HFD)-induced steatohepatitis and CKD share common pathogenesis involving peroxisome proliferator-activated receptor (PPAR)- $\alpha$ and $-\delta$. Elafibranor, a dual PPAR $\alpha / \delta$ agonist, can ameliorate the HFD-induced steatohepatitis. Nonetheless, the effects of HFD-induced CKD had not yet explored. This study investigated the effects of elafibranor (elaf) on the progression of HFD-induced CKD in mice. In vivo and in vitro renal effects were evaluated in HFD-elaf mice receiving 12 weeks of elafibranor (from $13^{\text {th }}$ to $24^{\text {th }}$ week of HFD feeding) treatment. In elafibranor-treated HFD mice, increased insulin sensitivity, reduced obesity and body fat mass, decreased severity of steatohepatitis, increased renal expression of PPAR $\alpha$, PPAR $\delta$, SIRT1, and autophagy (Beclin-1 and LC3-II) as well as glomerular/renal tubular barrier markers [synaptopodin (podocyte marker), zona occludin-1, and cubulin], reduced renal oxidative stress and caspase-3, and less urinary 8-isoprostanes excretion were observed. Aforementioned benefits of elafibranor were associated with low renal tubular injury and tubulointerstitial fibrosis scores, less albuminuria, low urinary albumin-to-creatinine ratio, and preserved glomerular filtration rate. Acute incubation of podocytes and HK-2 cells with elafibranor or recombinant SIRT1 reversed the HFD-sera-induced oxidative stress, autophagy dysfunction, cell apoptosis, barrier marker loss, albumin endocytosis, and reuptake reduction. Besides hepatoprotective and metabolic beneficial effects, current study showed that elafibranor inhibited the progression of HFD-induced CKD through activation of renal PPAR $\alpha$, $\operatorname{PPAR} \delta$, SIRT1, autophagy, reduction of oxidative stress, and apoptosis in mice with steatohepatitis.

\section{Introduction}

High-fat diet (HFD) intake and obesity have been associated with onset and progression of steatohepatitis and chronic kidney disease (CKD) [1-4]. In obesity, hemodynamic and morphological changes, together with other factors such as systemic inflammation, oxidative stress, and metabolic dyshomeostasis, may result in steatohepatitis and CKD and 
ultimately lead to cirrhosis and ESRD. Obesity was the second most highly predictive factor to predict end-stage renal disease (ESRD), even independent of diabetes and hypertension $[4,5]$.

Obesity-related CKD has been characterized by proteinuria, inflammation, and fibrosis [6]. Steatohepatitis and CKD share common pathogenic factors, incidences of both of them increased in severe obese patients $[1-3,5]$. HFD-fed mice is a widely used experimental model to induce obesity, CKD, and steatohepatitis [7].

The perioxisome proliferator-activated receptors (PPAR)$\alpha$ and $\operatorname{PPAR} \delta$ are crucial for the regulation of inflammation, oxidative stress, and metabolic dyshomeostasis in obese individuals with steatohepatitis $[8,9]$. Elafibranor is a novel dual PPAR $\alpha / \delta$ agonist to reduce impaired metabolism, inflammation, and fibrosis in obese patients and animals with steatohepatitis $[10,11]$.

$\operatorname{PPAR} \alpha$ agonists attenuate albuminuria and renal fibrosis in diabetic animals [12]. In diabetes mice, downregulated renal $\operatorname{PPAR} \delta$ expressions result in heavy albuminuria, renal inflammation, and fibrosis $[13,14]$. The advantage of dual $\operatorname{PPAR} \alpha / \delta$ agonist with respect to renal function was demonstrated by a lower risk of serum creatinine elevation with elafibranor (PPAR $\alpha / \delta$ agonist) user than fenofibrate (PPAR $\alpha$ agonist, $7.1 \%$ versus $17.2 \%$ ) user with obesity and steatohepatitis [12]. Sirtuin 1 (SIRT1) has been reported to have proautophagic, antioxidative stress, anti-inflammation, and antiapoptotic effects [14-19]. Both PPAR $\alpha$ and PPAR $\delta$ activation can stimulate SIRT1 expression [20, 21]. Hepatic and renal SIRT1 expressions are downregulated in high fat dietinduced obese animals with steatohepatitis and CKD [14, 15]. Renal SIRT1 activation attenuates diabetic albuminuria and ameliorates renal fibrosis $[16,17]$. Pharmacologic activation of SIRT1 can alleviate steatohepatitis and CKD in obese animals $[18,19]$.

Accordingly, this study evaluated the $\operatorname{PPAR} \alpha / \delta$-activated SIRT1-mediated molecular mechanism and effects of chronic elafibranor treatment on the progression of CKD in HFD-fed obese mice with steatohepatitis.

\section{Materials and Methods}

Additional information was included in the supplementary materials and methods (available here)

2.1. Animals. Male C57BL/6 mice (Jackson Laboratories, Bar Harbor, ME), 8 weeks old, were housed in temperature- and humidity-controlled rooms, kept on $12 \mathrm{~h}$ light/dark cycle, and provided unrestricted amounts of food and water. This study was approved by the Animal Experiments Committee of Yang-Ming University. Mice were provided with normal chow (NC, Laboratory Autoclavable Rodent Diet 5010) as NC group or a high-fat-diet (HFD, 60\% kcal in fat) as HFD groups. In concordance with previous reports [20, 21], CKD including albuminuria (renal damage) and decreased creatinine clearance (poor renal function) were observed in HFD-24w mice [mice feeding with 24 weeks of HFD] in preliminary experiments $(n=3)$.
The groups (Figure 1(a)) of C57BL/6 mice included the following: $N C-24 w$ group $(\mathrm{n}=5) / H F D-24 w$ group $(\mathrm{n}=8)$ continuously fed NC/HFD for 24 weeks were administered vehicle for 12 weeks from $13^{\text {th }}$ to $24^{\text {th }}$ week of NC/HFD feeding; HFD-elaf group $(n=8)$ continuously fed HFD for 24 weeks were administered by oral gavage elafibranor $(3 \mathrm{mg} / \mathrm{kg} /$ day $)$ for 12 weeks from $13^{\text {th }}$ to $24^{\text {th }}$ week of HFD feeding, at which time steatohepatitis, albuminuria, and a decrease in GFR developed. This dose of elafibranor ( $3 \mathrm{mg} / \mathrm{kg} /$ day) has been demonstrated previously to decrease the progression of steatohepatitis $[10,12]$.

\subsection{Blood Pressure, Metabolic Demands, and Renal Function.} The mouse was placed in a metabolic cage and had free access to diet and water. Then, 24-hour urine sample was collected at 3 consecutive days, and the average of 3-day urine was calculated. The supernatant of centrifuged 3day urine samples was used for various analyses, including albumin, creatinine, and 8-isoprostane [marker of oxidative stress]. Urinary albumin-to-creatinine ratio (ACR) was calculated as $\mathrm{ACR}=$ urine albumin/urine creatinine $(\mu \mathrm{g} / \mathrm{mg})$. One day later, mouse GFR was measured after a single injection of FITC-inulin. The GFR was calculated using a two-compartment model of two-phase exponential decay. All above measurements were undergone in Taiwan Mouse Clinic (National Phenotyping and Drug Testing Center) on week 24 of the feeding regimen for HFD-24w/HFD-elaf/NC24w groups.

Mouse GFR was measured by single injection of FITCinulin clearance as described previously, modified to minimize plasma volume. The GFR was calculated using a twocompartment model of two-phase exponential decay. Briefly, dialyzed FITC-inulin $(3.74 \mu \mathrm{l} / \mathrm{g}$ body wt, Sigma-Aldrich, Inc., St. Louis, MO) was injected retro-orbitally under light anesthesia induced using isoflurane (Baxter Pharmaceutical Products, Deerfield, IL). The anesthesia lasted 20 s. Approximately $20 \mu \mathrm{l}$ of blood was collected via the saphenous vein at $10,35,55$, and $75 \mathrm{~min}$ after injection of FITC-inulin for the determination of FITC concentration.

2.3. Basal Measurements. Glucose tolerance test (GTT) was performed after overnight $(16 \mathrm{~h})$ fasting by intraperitoneal injection of D-glucose $(2 \mathrm{mg} / \mathrm{g}$ body weight, Sigma-Aldrich, Inc., St. Louis, MO). Blood glucose was measured at 0, 30, 90, and 120 minutes using blood obtained by tail nicking using a One Touch glucometer (One Touch Ultra2, Life Scan, Johson\&Johson, USA). All animals continued their initial feeding regimen until scarification. Under anesthesia, 2 days after stabilization and overnight fasting, heparinizedblood (from the inferior-vena-cava, abdominal-aorta, and heart-chamber) and the liver/kidney were collected and weighted.

2.4. Serum and Tissue Metabolic and Inflammatory Profiles. Serum biochemistry data, triglyceride, insulin, $\mathrm{TNF} \alpha$, and IL-6, caspase-3/7 activity as well as renal IL-6/TNF- $\alpha$ levels, myeloperoxidase (MPO) and SIRT1/caspase 3 activities, and hepatic SIRT1 activity were measured. 


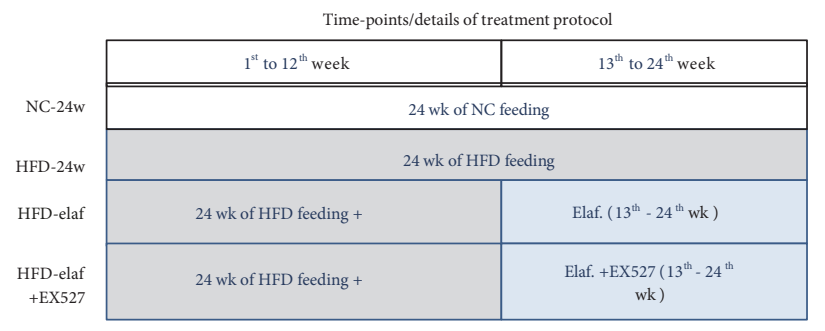

(a)

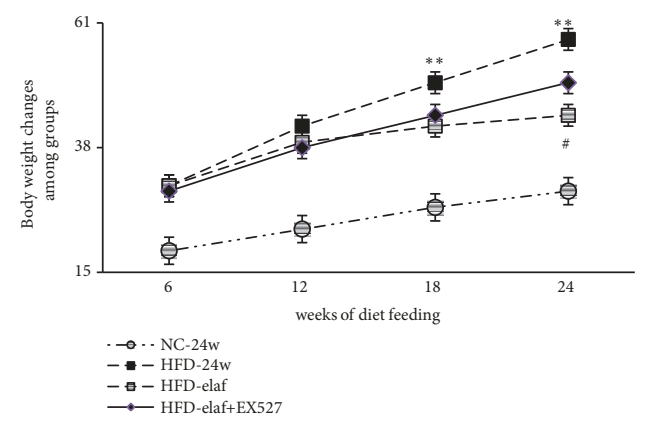

(b)

Representative image and bar graphs of H-E-stained liver section for nonalcoholic fatty liver disease activity score (NAS) , x10, scale bar $100 \mu \mathrm{m}$

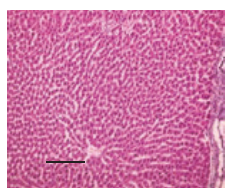

NC-24w

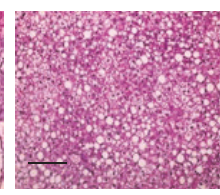

HFD-24w

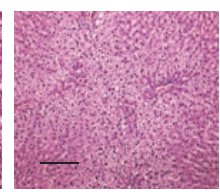

HFD-elaf

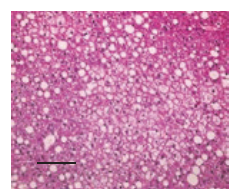

HFD-elaf-EX527

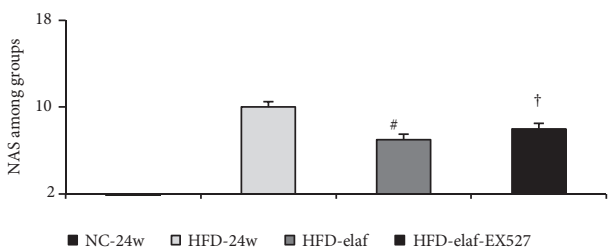

(c)

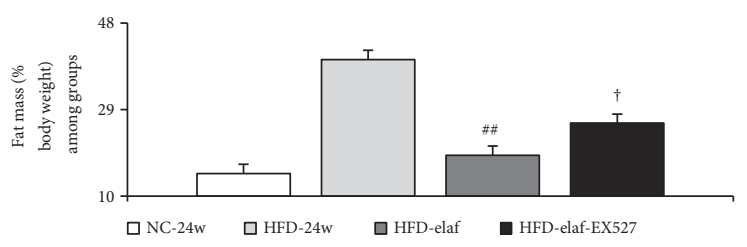

(d)

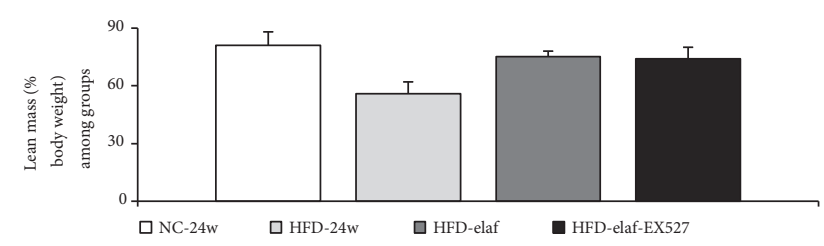

(e)

FIGURE 1: Chronic dual PPAR $\alpha / \delta$ agonist, elafibranor, treatment improved severity of adiposity in HFD mice with steatohepatitis. (a) Treatment protocol; (b) growth curves of HFD or NC mice: elafibranor decreased the trend of increased body weight, which can be abolished by concomitant SIRT1 inhibitor (EX527) treatment. Food and water consumption was estimated by daily observation at the time of feeding, and body weight was recorded every 2 weeks. (c) Representative image and bar graphs of H-E stain for hepatic steatosis: elafibranor decreased the severity of steatohepatitis; (d-e) whole body fat and lean mass of mice: elafibranor significantly reduced the fat mass of HFD mice. $*, * * p<0.05,0.01$ versus NC-group. $\stackrel{\#, \#}{\text { p }}<0.05,0.01$ versus HFD-group; $\dagger p<0.05$ versus HFD-elaf group.

2.5. Histologic Analysis. Nonalcoholic fatty liver disease activity score (NAS) was measured by $\mathrm{H}$-E-stained liver section. The $\mathrm{H}$-E and periodic acid-Schiff(PAS)-stained renal section was evaluated to score the renal tubular damage and tubulointerstitial fibrosis. With an ApopTag Peroxidase In Situ Apoptosis Detection Kit (Chemicon, CA, USA), glomerular and tubules cells undergoing apoptosis were calculated.

2.6. Renal Electron Microscopic and Immunofluorescence Analysis. Then, the isolated membranes and autophagosomes on the ultrathin section in the proximal renal tubule of kidney were calculated by electron microscopy at $1,200 \mathrm{x}$ magnification. Meanwhile, each slide was evaluated for the numbers of cubulin/synaptopodin $(+)$ cells per $1 \mathrm{~mm}^{2}$ in the FITC images.

2.7. Protein and $m R N A$ Measurements. Mouse podocytes and HK-2 cells were purchased from the CELPROGEN (3914 Del, Amo Blvd, Suite 901, Torrance, CA 90503) and Bioresource Collection and Research Center (BCRC, Hsin-Chu, Taiwan). Then, various proteins and mRNAs (with primers listed in Table 1) were measured in podocytes/HK-2 cell lysates, glomerular and tubular fractions of renal homogenates.

2.8. Roles of SIRT1-Autophagy on Elafibranor-Related Effects on HFD-Sera-Pretreated Podocytes and HK-2 Cells. HFD/ NC-sera were obtained from NC-24w and HFD-24w mice. To mimic the impacts of circulating factors of HFD mice on abnormalities of renal microenvironment, various measurements were undertaken in 10\% HFD-sera-pretreated podocytes/HK-2 cells. Significantly, 10\% HFD-sera incubation suppressed the SIRT1 activity in cell lysates of podocytes/HK-2 cells. A preliminary dose-finding experiment revealed that, among different concentrations $(5,10$, 15 , and $30 \mu \mathrm{M})$ of elafibranor, maximal stimulation of SIRT1 activity on HFD-sera-pretreated cells was noted at $15 \mu \mathrm{M}$ of elafibranor. Meanwhile, siSIRT1 was transfected into cells and maximal blockade of elafibranor-activated SIRT1 activity was noted at $100 \mu \mathrm{M}$.

Meanwhile, we found that $\operatorname{rSIRT1}(300 \mu \mathrm{M})$ had similar effects as elafibranor $(15 \mu \mathrm{M})$ to reverse HFD-sera-suppressed SIRT1 activity in cells. To examine the SIRT1-mediated effects of elafibranor on autophagy, HFD-sera-pretreated cells were 
TABLE 1: Primer of various genes measured in this study.

\begin{tabular}{lcr}
\hline Name of gene & Sequence of sense primer (5-3) & Sequence of anti-sense primer (3-5) \\
\hline PPAR $\alpha$ & 5-ATGCCAGTACTGCCGTTTTC-3 & 5-TTGCCCAGAGATTTGAGGTC-3 \\
\hline PPAR $\delta$ & 5-CCCTTCATCATCCACGACATT-3 & 5- TGGACTGGCAGCGGTAGAAC -3 \\
\hline Sirt1 & 5-GCAACAGCATCTTGCCGAT-3 & 5- GTGCTACTGGTCTCACTT -3 \\
\hline Sirt3 & 5-CAGCAACCTTCAGCAGTA-3 & 5-CCGTGCATGTAGCTGTTA-3 \\
\hline AMPK $\alpha 1$ & 5-CAGGGACTGCTACTCCACAGAGA-3 & 5-CCTTGAGCCTCAGCATCTGAA-3 \\
\hline AMPK $\alpha 2$ & 5-CAACTGCAGAGAGCCATTCACTT-3 & 5-GGTGAAACTGAAGACAATGTGCTT-3 \\
\hline Beclin-1 & 5- AATCTAAGGAGTTGCCGTTATAC-3 & 5-CCAGTGTCTTCAATCTTGCC-3 \\
\hline LC3-II & 5- GATGTCCGACTTATTCGAGAGC-3 & 5- TTGAGCTGTAAGCGCCTTCTA-3 \\
\hline NADPH oxidase subunits p22phox & 5- GCGGTGTGGACAGAAGTACC -3 & 5- CTTGGGTTTAGGCTCAATGG -3 \\
\hline Nox-4 & 5-ACAGTCCTGGCTTACCTTCG -3 & 5-TTCTGGGATCCTCATTCTGG-3 \\
\hline ZO-1 & 5-CCACCTCTGTCCAGCTCTTC-3 & 5-CACCGGAGTGATGGTTTTCT-3 \\
\hline Cubulin & 5- GCTCAACCTCCATTCAATCATA-3 & 5-GTGCAATCTGTGCTGCTT-3 \\
\hline 18S & 5- GTAACCCGTTGAACCCCATT-3 & 5-CCATCCAATCGGTAGTAGCG-3 \\
\hline
\end{tabular}

incubated with bafilomycin A1 (BAF, 100ng/mL, a blocker of autophage flux) concomitantly with elafibranor $(15 \mu \mathrm{M})$ or rSIRT1 $(300 \mu \mathrm{M})$. For the following in vitro experiments, vehicle (V), elaf, elaf+siSIRT1, rSIRT1, rSIRT1+BAF group in either HFD-sera- or NC-sera-pretreated cells were included.

2.9. Albumin Endocytosis or Albumin Reuptake of HFDSera-Pretreated Podocytes and HK-2 Cells. For albumin endocytosis and albumin reuptake experiments, pretreated podocytes/HK-2 (1x10 ${ }^{5}$ cells) cells were incubated with $1.5 \mathrm{mg} / \mathrm{ml}$ human FITC-albumin (MP Biomedicals, Santa Ana, CA) in Ringer solution at either $4^{\circ} \mathrm{C}$ or $37^{\circ} \mathrm{C}$ for 1 hour. Afterward, for spectrofluorometric measurements, podocytes/HK-2 were lysed in $20 \mathrm{mM}$ MOPS with $0.1 \%$ Triton X-100. FITC-fluorescence was measured using an excitation wavelength of $490 \mathrm{~nm}$ and an emission wavelength of $540 \mathrm{~nm}$ by a fluorescence plate reader (Synergy HT; Biotek Instruments, Winooski, VT). The amount of protein in the lysates was measured using the bicinchoninic acid (BCA) assay (Pierce, Rockford, IL) and the amount of cell associated FITC-albumin was expressed as FITC-albumin $(\mathrm{mg} / \mathrm{mL})$ to compare degree of albumin endocytosis/reuptake between groups.

2.10. SIRT1-Autophagy Protein and mRNA Levels in Cultured Podocyte and HK-2 Cells. Notably,same protocol in albumin endocytosis (podocyte) and reuptake (HK-2) experiments was used to prepare cells for this part. After supernatants were collected for 8 -isoprostane, caspase $3 / 7$ activity was measured by the ELISA and luminescent substrate assay (CaspaseGlo assay; promega). Proteins and mRNAs were extracted, and cell lysates were used for SIRT1 activities measurement by Biomol SIRT1 fluorescence assay kit (AK-555; Biomol, Farmingdale, NY, USA).

Specifically, for calculation of autophagy flux index, cells were treated with $125 \mathrm{nM}$ of Bafilomycin A1 [inhibitors for maturation step of autophagosome including lysosomal enzyme activity and fusion of autophagosomes with lysosomes], in DMSO 2 hours before harvest to obtain cell lysates for measurement of LC3-II protein expression. Then, autophagy flux index was calculated by the formula [autophagy flux index=LC3-II (indicator of autophagosomes formation) expression levels with Bafilomycin A1 (100ng/mL)/LC3-II expression levels without BafilomycinA1]. LC3-II expression was normalized by its GADPH expression level.

2.11. Apoptotic and Barrier Markers (+) Podocytes and HK2 Cells. Cells were stained with Annexin V and 7-aminoactinomycin D (7-AAD; BioLegend, San Diego, CA) and analyzed by flow cytometry with FCSCanto II (BD Biosciences, Mississauga, ON). For immunofluorescence (IF) staining, cells were fixed in paraformaldehyde followed by permeabilization with $0.025 \%$ digitonin in PBS. After washing, the cells were subsequently incubated at RT with synaptopodin/cubulin/LC3-II antibodies, FITC-conjugated secondary antibody. After washing with PBS, optical section data for \% of synaptopodin/cubulin/LC3-II (+) area on each slide were evaluated.

2.12. Statistical Analysis. Results are presented as means \pm SD. Data were analyzed by ANOVA and Student-Newman-Keuls tests for multiple comparisons or by Student's $t$-test for unpaired data between two groups. Statistical significance was accepted at the $P<0.05$ level.

\section{Results}

3.1. Chronic Elafibranor Treatments Improve Metabolic Profiles in HFD Mice. In comparison with NC-24w group, 24 weeks of HFD feeding induced hyperglycemia, hyperinsulinemia, abnormal GTT, higher homeostasis model assessment-insulin-resistance (HOMA-IR) index, more food consumption, greater incremental trend of body weight, higher serum/hepatic triglyceride level, and higher whole body fat mass were observed in HFD-24w group (Figures 1, 2(b), and 2(e), Table 2). Nonetheless, water consumption 


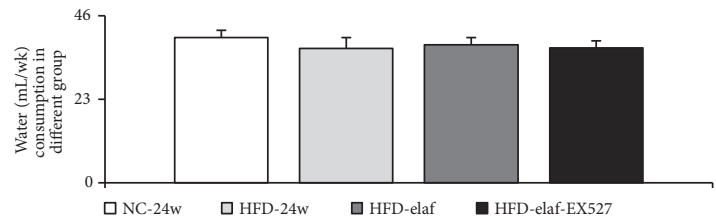

(a)

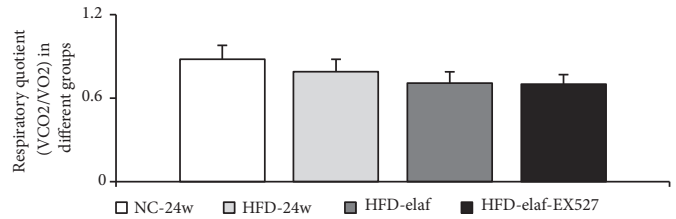

(c)

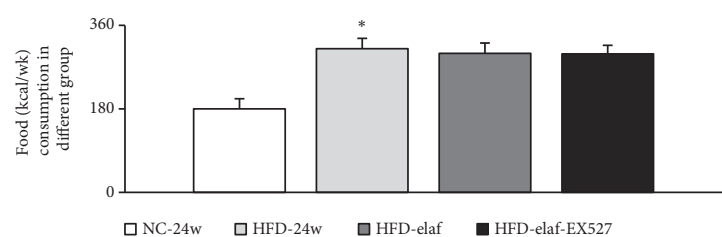

(b)

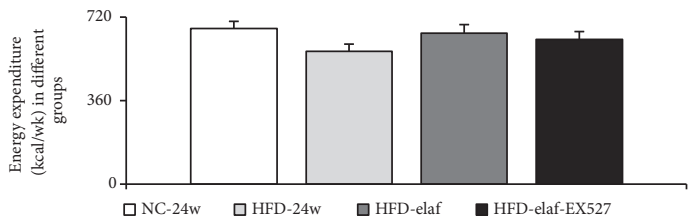

(d)
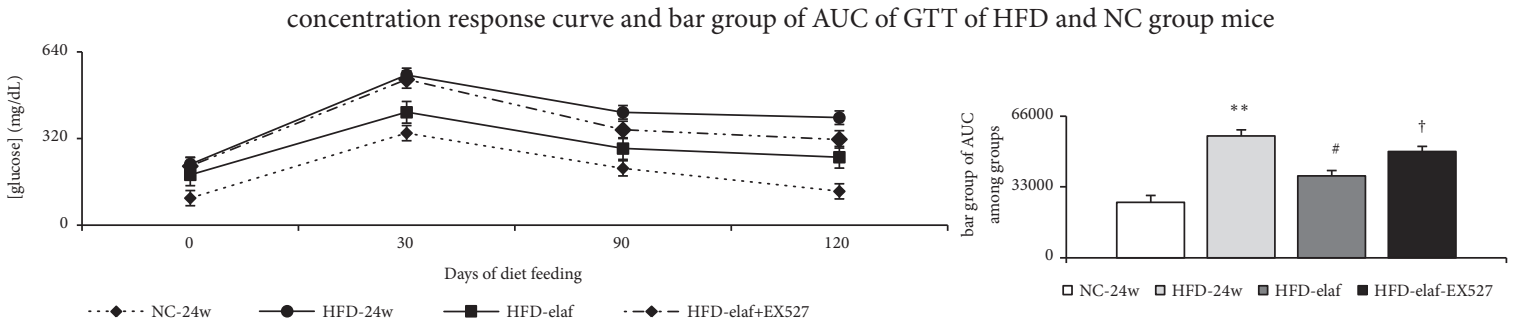

(e)

FIGURE 2: Chronic elafibranor treatments increased insulin sensitivity of HFD mice with steatohepatitis. Elafibranor had no effects on water $((\mathrm{a}), \mathrm{mL} / \mathrm{wk})$ and food ((b), kcal/wk) consumption; parameters of metabolic demands, respiratory quotient (c)/energy expenditure (d); (e) concentration-response curve and area under curve (AUC) of glucose tolerance test (GTT): elafibranor significantly improved the GTT, which was abolished by concomitant SIRT1 inhibitor (EX527) treatment. $*, * * p<0.05,0.01$ versus NC-group; \#p $<0.05$ versus HFD-group; $\dagger \mathrm{p}<0.05$ versus HFD-elaf group.

TABLE 2: Effect of chronic elafibranor (elaf) treatment on the inflammatory profiles of HFD mice with steatohepatitis and CKD.

\begin{tabular}{|c|c|c|c|}
\hline & NC-24w & HFD-24w & HFD-elaf \\
\hline Kidney weight (mg) & $356 \pm 10$ & $423 \pm 9 *$ & $318 \pm 34$ \\
\hline [triglyceride, TG, mg/dL] & $57 \pm 3.9$ & $299 \pm 28 *$ & $211 \pm 14^{\#}$ \\
\hline Hepatic TG levels (mg/g) & $98 \pm 7$ & $240 \pm 18 *$ & $200 \pm 10^{\#}$ \\
\hline [fasting glucose] (mg/dL) & $116 \pm 13$ & $243 \pm 28 *$ & $203 \pm 9^{\#}$ \\
\hline [fasting Insulin] (ng/mL) & $1.9 \pm 0.2$ & $6.9 \pm 0.85 *$ & $5.2 \pm 0.8$ \\
\hline $\begin{array}{l}\text { Homeostasis model assessment-insulin-resistance } \\
\text { (HOMA-IR) index }\end{array}$ & $3.8 \pm 0.4$ & $28.8 \pm 6.4 *$ & $18.1 \pm 1.1^{\#}$ \\
\hline [Aspartate aminotransferase] (AST, U/L) & $40.9 \pm 1.6$ & $117.3 \pm 20.1 *$ & $79.5 \pm 4.3^{\#}$ \\
\hline [Alanine aminotransferase] (ALT, U/L) & $46.8 \pm 10.3$ & $183.2 \pm 6.8 *$ & $104.2 \pm 13.7^{\#}$ \\
\hline$[\mathrm{IL}-6, \mathrm{pg} / \mathrm{mL}]$ & $144 \pm 18$ & $223 \pm 35 *$ & $168 \pm 9^{\#}$ \\
\hline Kidney IL-6 (pg/mg protein) & $1.8 \pm 0.4$ & $7 \pm 0.8 *$ & $6.2 \pm 1.9$ \\
\hline$[\mathrm{TNF} \alpha, \mathrm{pg} / \mathrm{mL}]$ & $12 \pm 5$ & $40 \pm 11 *$ & $29 \pm 8^{\# \#}$ \\
\hline Renal TNF $\alpha$ (pg/mg protein) & $4 \pm 0.8$ & $16 \pm 2.3 *$ & $9 \pm 1.1^{\#}$ \\
\hline Renal MPO activity (U/g) & $8.6 \pm 1.5$ & $51 \pm 20 *$ & $31 \pm 4^{\#}$ \\
\hline Renal caspase- 3 activity (pmol/ $\mu$ g protein) & $35 \pm 1$ & $90 \pm 5 *$ & $72 \pm 3^{\#}$ \\
\hline
\end{tabular}

NC-24w/HFD-24w: mice receiving 24-week high-fat diet (HFD) or normal chow (NC) feeding and vehicle treatment; NC-elaf/HFD-elaf: HFD- or NC-fed mice receiving 12 -week elafibranor treatment from $13^{\text {th }}$ to $24^{\text {th }}$ week of either HFD or NC feeding; HOMA-IR: calculated as ([fasting glucose] $\times$ [fasting insulin])/58.32.*,**p<0.05,0.01 vs. NC-group; $\#$ \#\# $\mathrm{p}<0.05,0.01$ vs. HFD-group. 


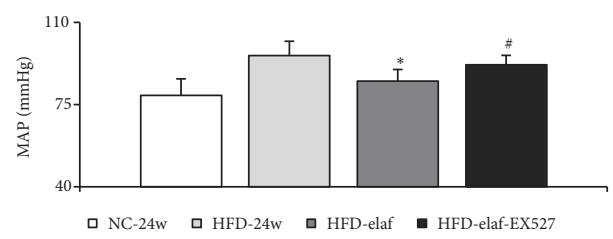

(a)

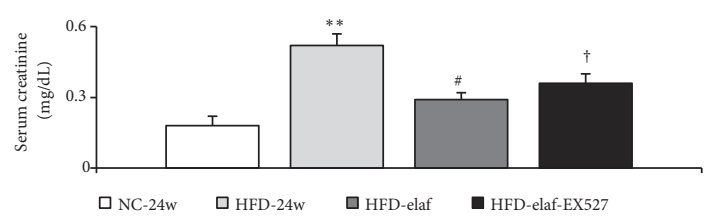

(b)

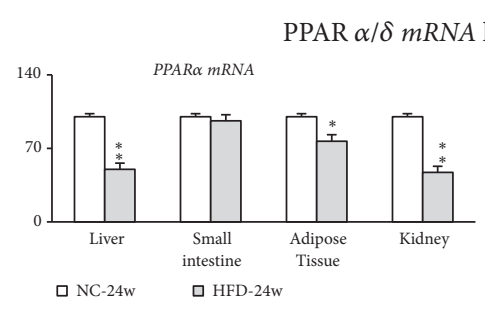

(c)

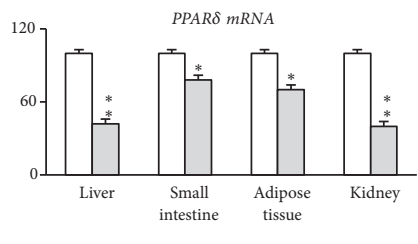

)

energy nutrient sensor markers $m R N A$ levels in different tissue

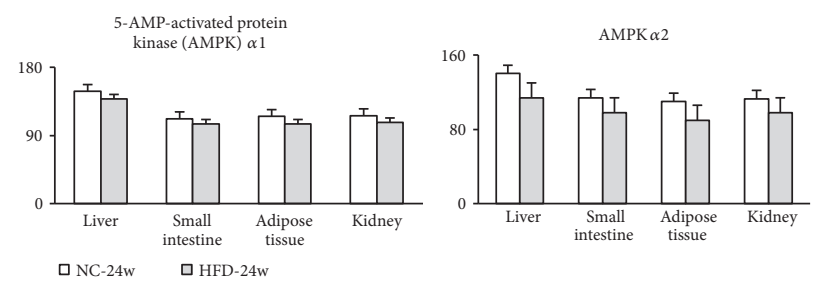

(d)

SIRT1 / SIRT3 mRNA levels in different tissue

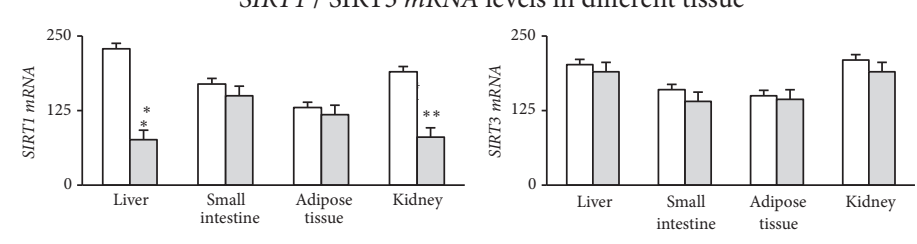

(e)

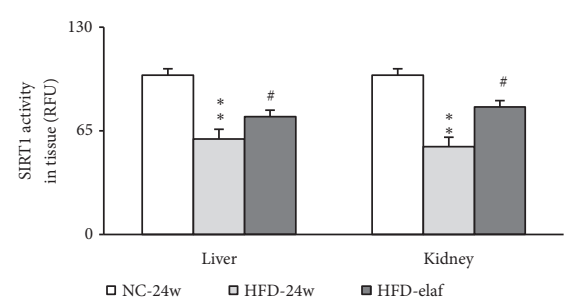

(f)

FIGURE 3: Chronic elafibranor treatments improved the renal function through the activation of renal SIRT1 in HFD mice with steatohepatitis and CKD. (a) Mean arterial blood pressure (MAP, mmHg); (b) serum creatinine (mg/dL); (c) PPAR $\alpha / \delta$; and (d-e) energy nutrient sensor markers $m R N A$ levels (\%/18S) in main target tissue of HFD mice; (f) liver and renal SIRT1 activity (RFU, relative fluorescence units). $*, * * p<0.05,0.01$ versus NC-group; $\# \mathrm{p}<0.05,0.01$ versus $\mathrm{HFD}$-group; $\dagger \mathrm{p}<0.05$ versus $\mathrm{HFD}$-elaf group.

and metabolic demands [respiratory quotient (average whole body $\mathrm{CO}_{2}$ production $/ \mathrm{O}_{2}$ consumption) and energy expenditure] were not different between NC group and HFD-group (Figures 2(a), 2(c), and 2(d)).

In HFD group, the beneficial effects of elafibranor with respect to hepatic steatosis, whole body fat mass, and GTT were counteracted by EX527 (a specific SIRT1 inhibitor), but not in body weight changes (Figures $1(\mathrm{~b})-1(\mathrm{~d})$ and $2(\mathrm{e})$, Table 2).,

\subsection{Characteristics of CKD in HFD Mice with Steatohepatitis.} The CKD findings in HFD mice include increased serum creatinine, increased albuminuria, urine ACR, decreased GFR, decreased renal PPAR $\alpha / \delta$, and SIRT1 expressions/activity (Figures 3(b), 3(c), 4(a), and 5(a)-5(c)).

Although no difference in water consumption and urine output was observed, significantly, increased kidney weight, renal MPO activity, and urinary 8-isoprostanes excretion were observed in HFD-group with CKD compared with that in the NC group (Table 2, Figures 2(a), 5(d), and 5(e)).

\subsection{Elafibranor Normalizes Hepatic and Renal SIRT1 Expres-} sion in HFD Mice with Steatohepatitis and CKD. In the HFD group, a general reduction in liver, adipose tissue, and renal PPAR $\alpha$ and PPAR $\delta$ expression than in the NC group was observed (Figure 3(c)). In our study, the expression of other energy and nutrient sensors (AMPK $\alpha 1 / 2$ and SIRT3) in the liver, small intestine, adipose tissue, and kidney was not different between the HFD-group and the NC-group (Figures $3(\mathrm{~d})-3(\mathrm{f}))$.

A similar decreasing trend of PPAR $\alpha$, PPAR $\delta$, and SIRT1 expression in the liver and kidney was observed in HFD mice with steatohepatitis and CKD (Figures 3(c), 3(e), 3(f), and 4(a)). Nonetheless, the expression of SIRT1 in the small intestine and adipose tissue was not different between the NC group and the HFD group. Remarkably, simultaneous activation of hepatic and renal PPAR $\alpha$ and PPAR $\delta$ by preventive or therapeutic elafibranor treatment restored hepatic and renal SIRT1 expression in the HFD group. Elafibranor-related decrease in severity of steatohepatitis was accompanied by an improvement of CKD (Figures 1(c), 3(b), 5(a), and 5(b)). In the electron microscopic images of renal section, a decrease in double membrane structure and autophagosome was observed in the proximal renal tubule of kidney of HFD mice compared to the NC group, which was increased after elafibranor treatment (Figures 4(b) and 4(c)).

\subsection{Elafibranor Improves Inflammatory and Apoptotic Profiles} in HFD Mice with Steatohepatitis and CKD. In HFD mice, the high serum/renal TNF $\alpha$ levels, serum caspase 3/7, renal Tunnel stain-assessed apoptotic activity, serum IL-6, serum AST, and ALT were significantly suppressed by elafibranor 


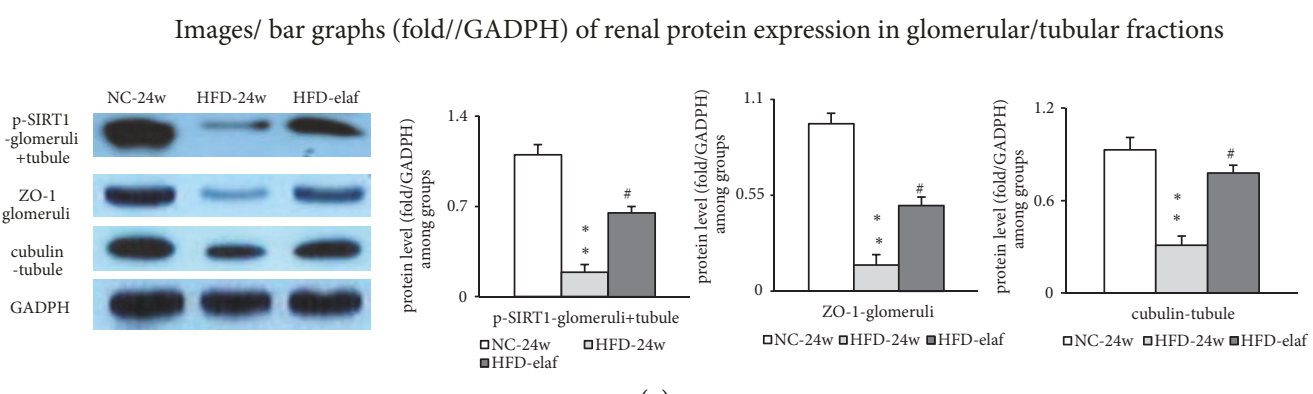

(a)

representative electron micrographs (b)/ bar graph (c) of autophagosome in kidney of HFD mice

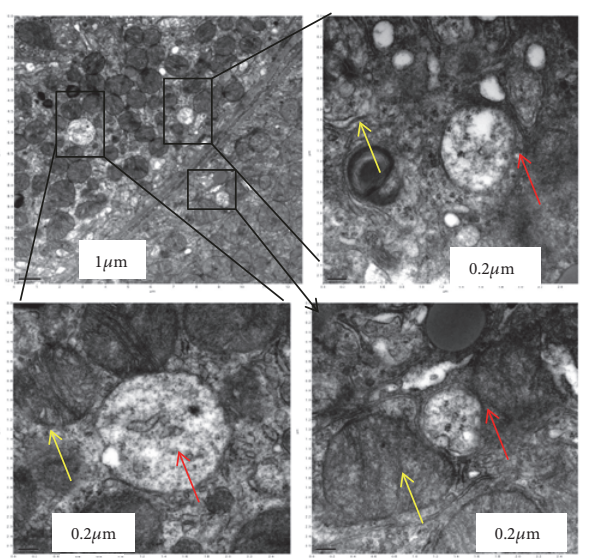

(b)

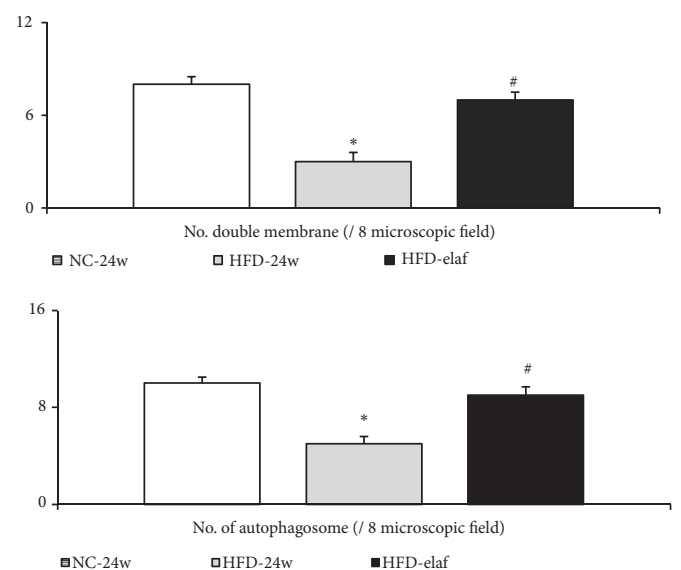

(c)

FIGURE 4: Increased SIRT1 expression was accompanied by restoration of renal glomerular and tubular barrier protein expression in elafibranortreated HFD mice with CKD. (a) Representative image/bar graph of various protein expression in glomeruli/tubular fraction. Representative electron microscopy (EM) images (1,200x) (b)/bar graphs (c) of average number of double membrane (yellow arrow) and autophagosome (red arrow) in eight randomly selected fields in the proximal renal tubule of kidney of HFD mice, decrease in the number of double membrane and autophagosome was restored by elafibranor treatment. $*, * * p<0.05,0.01 ;{ }^{*} \mathrm{p}<0.05$ versus HFD-group.

treatment, and the effect was reversed by EX-527 (Table 2 and Figures 5(f) and 5(g)). Nonetheless, renal IL-6 levels were not different between HFD mice with and without elafibranor treatment.

\subsection{Renal SIRT1 Activation by Elafibranor Is Accompanied} by Normalization of Renal Barrier Markers in HFD Mice. Decreased renal p-SIRT1 expressions were accompanied by the reduction of the expression of glomerular [synaptopodin (marker of podocyte)/ZO-1] and tubular [cubulin] barrier markers in the HFD-group (Figures 4(a), and 6(a)-6(d)).

Notably, chronic elafibranor treatment partially restored the aforementioned renal barrier markers expression in HFD group (Figures 4(a), and 6(a)-6(d)). In the HFD group, the lower renal PPAR $\alpha / \delta$ expression was associated with less autophagy (Beclin-1 and LC3-II)/barrier (synaptopodin, cubulin, and ZO-1) markers, and more oxidative-stress (p22phox and Nox-4) markers relative to the NC group (Table 2, Figures 3(c), 4(b), and 6).

3.6. Elafibranor Treatment Inhibits the Progression of CKD in HFD Mice with Steatohepatitis. In comparison with HFD24w mice, decreased albuminuria (Figure 5(a)) and improved
GFR (Figure 5(c)), reduced tubular injury and tubulointerstitial fibrosis scores (Figure $5(\mathrm{~g})$ ), were associated with the restoration of renal PPAR $\alpha / \mathrm{PPAR} \delta / \mathrm{SIRT} 1 /$ autophagy (increased Beclin-1/LC3-II) and barrier (synaptopodin/ZO1/cubulin) markers (Figures 3(c)-3(f), 4(a), and 6), the suppression of renal oxidative stress [p22phox and Nox-4, MPO activity and urinary 8 -isoprostane excretion] (Figures $5(\mathrm{e})$ and 6(c)-6(e) and Table 2), and reduction of renal apoptosis in HFD-elaf mice (Table 2, Figures 5(e), 5(f), and 6(c)-6(e)); these effects were inhibited by concomitant EX527 (SIRT1 inhibitor) treatment. These results suggest that SIRT1 mediated the renal protective effects of chronic elafibranor treatment in HFD mice by activation of PPAR $\alpha$ and PPAR $\delta$ (Figures 4(a), 6(c), and 6(d)).

3.7. In Vitro Effects of Elafibranor in HFD-Sera-Pretreated Podocyte/HK-2 Cells. In HFD-sera-pretreated podocytes, compared with NC-sera-stimulated cells, less anti-inflammatory (SIRT1) activity, decreased autophagy (low Beclin1/LC3-II and autophagy flux index) level, downregulated barrier protein (synaptopodin/ZO-1) expression, impaired albumin endocytosis, and increased oxidative stress (8isoprotane, p22phox and Nox-4) and apoptosis (caspase 3/7 activity) were noted. These effects were reversed by elafibranor administration (Table 3, Figure 7). 


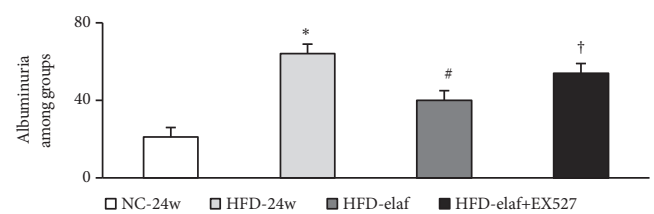

(a)

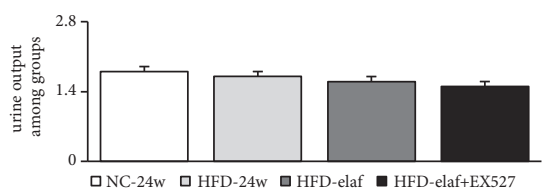

(d)

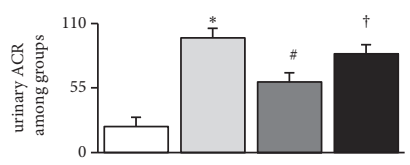

(b)

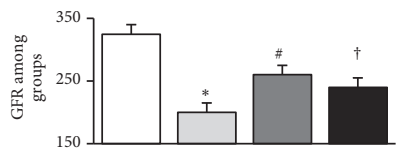

(c)

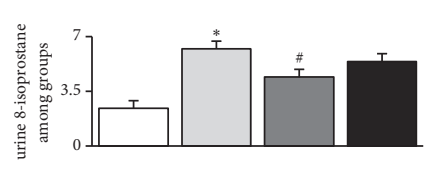

(e)

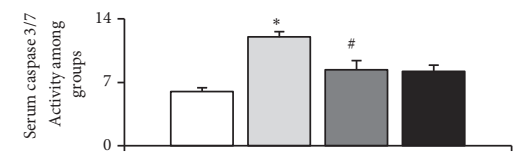

(f) representative images/bar graphs of different staining of renal tissue
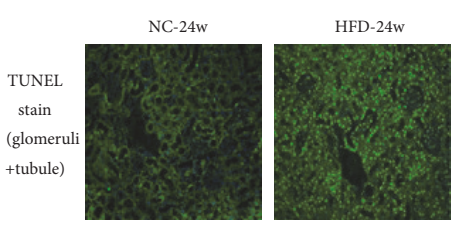
HFD-elaf
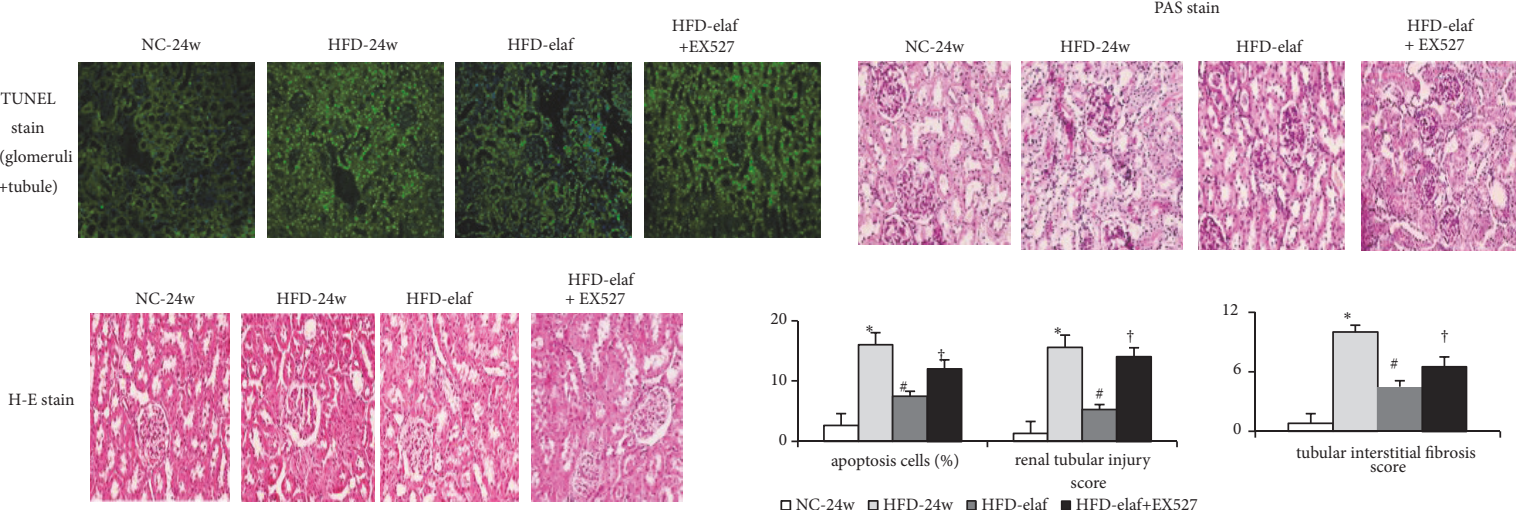

(g)

FIGURE 5: Chronic elafibranor treatment suppressed the progression of CKD through the inhibition of renal oxidative stress/apoptosis in HFD mice with steatohepatitis. (a) Albuminuria (urine albumin, $\mu \mathrm{g} / \mathrm{day}$ ); (b) urinary albumin-to-creatinine ratio (ACR, albumin $\mu \mathrm{g} / \mathrm{mg}$ of creatinine); (c) glomerular filtration rate (GFR, $\mu \mathrm{L} / \mathrm{min})$; (d) urine output; (e) urine 8-isoprostane (ng/mg cr) secretion; (f) serum caspase 3/7 activity; (g) histologic images/bar graphs of severity of renal injury. Renal tubular damage was assessed using a tubular damage score including atrophy and flattening of proximal tubule epithelial cells, and tubular dilation: $0=$ normal; $1=<20 \% ; 2=20$ to $40 \% ; 3=40$ to $60 \%$; $4=60$ to $80 \%$; and $5=80 \%$. The features of tubulointerstitial fibrosis include tubular atrophy/dilatation, presence of mononuclear inflammatory cells, widening of interstitial spaces with deposition of extracellular matrix, interstitial cell proliferation and wrinkling or thickened tubular basement membrane. $* p<0.05$ versus NC-group; $\# p<0.05,0.01$ versus HFD-group; $\uparrow p<0.05$ versus HFD-elaf group.

The beneficial effects of elafibranor were eliminated following siRNA targeting SIRT1. Interestingly, recombinant SIRT1 (rSIRT1) has similar effects (anti-inflammation, antiapoptosis, and autophagy activation) as elafibranor. Particularly, both elafibranor+siSIRT1 and rSIRT1-related effects can be blocked by Bafilomycin (blocker of autophagy flux) con-incubation. These results indicate that SIRT1-autophagy cascade plays a pivotal role in elafibranor-related effects of podocytes.

In cultured HK-2 cells monolayer, the effects of abovementioned treatment were similar to those in cultured podocytes. In particular, the changes in the renal tubular barrier marker, cubulin, were similar as changes in glomerular barrier markers (synaptopodin/ZO-1) in podocytes (Table 3 and Figure 8).

\section{Discussion}

Chronic elafibranor treatment inhibits the progression of HFD-induced CKD in mice in this study. Reduction in
GFR and increasing albuminuria are initial markers for the detection of the progression to ESRD [22]. In this study, the renoprotective effects of chronic elafibranor treatment, including preserved GFR and decreased albuminuria, were observed in HFD-induced obese mice with steatohepatitis.

In PPAR $\alpha$ knockout and diabetic mice, heavy albuminuria is associated with significant renal inflammation, apoptosis, and fibrosis [13, 23]. In renal tubular cells, PPAR $\alpha$ activation protects cells from gentamicin-induced oxidative stress and apoptosis [24]. Both PPAR $\alpha$ and PPAR $\delta$ are highly expressed in kidney $[11,13,24]$. Activation of PPAR $\delta$ ameliorates tubulointerstitial inflammation in mice with proteinuric kidney disease [25]. PPAR $\delta$ activation protects cardiomyoblasts from oxidative stress-induced apoptosis [26]. Accordingly, it is reasonable that elafibranor, through renal PPAR $\alpha$ and PPAR $\delta$ activation, improves CKD through inhibition of renal oxidative stress, inflammation, fibrosis, and apoptosis in HFD-induced obese mice with steatohepatitis in our study.

In fact, the renoprotective effects of 12 weeks of PPAR $\alpha$ agonist treatment had been reported in HFD-fed obese mice 


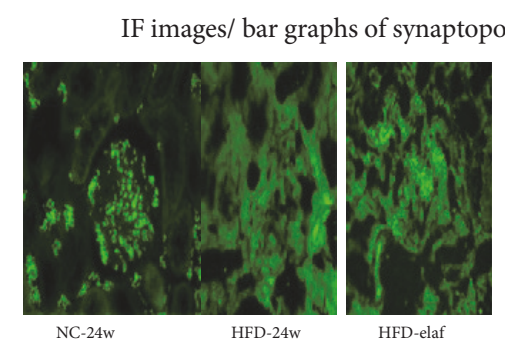

(a)

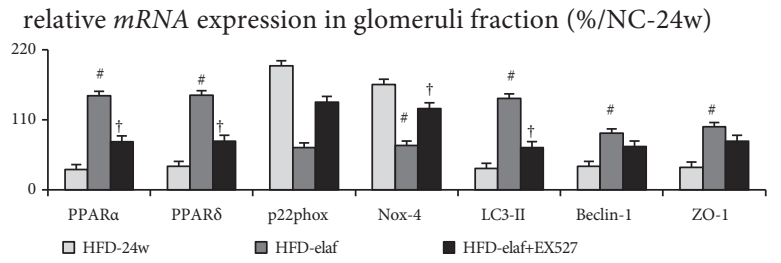

(c)
IF images/ bar graphs of renal cubulin expression in tubules
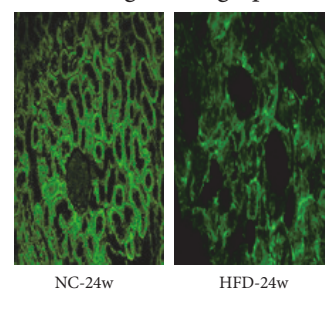

HFD-24w

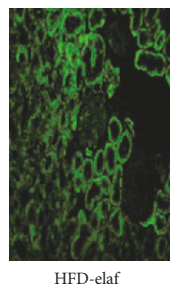

HFD-elaf

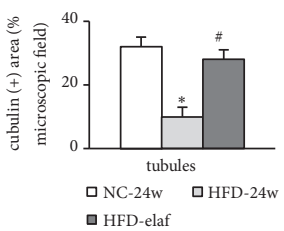

(b)

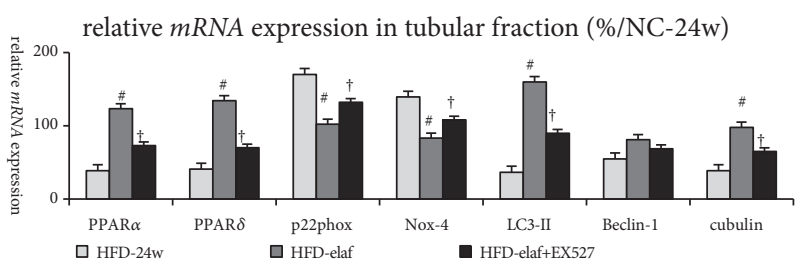

(d)
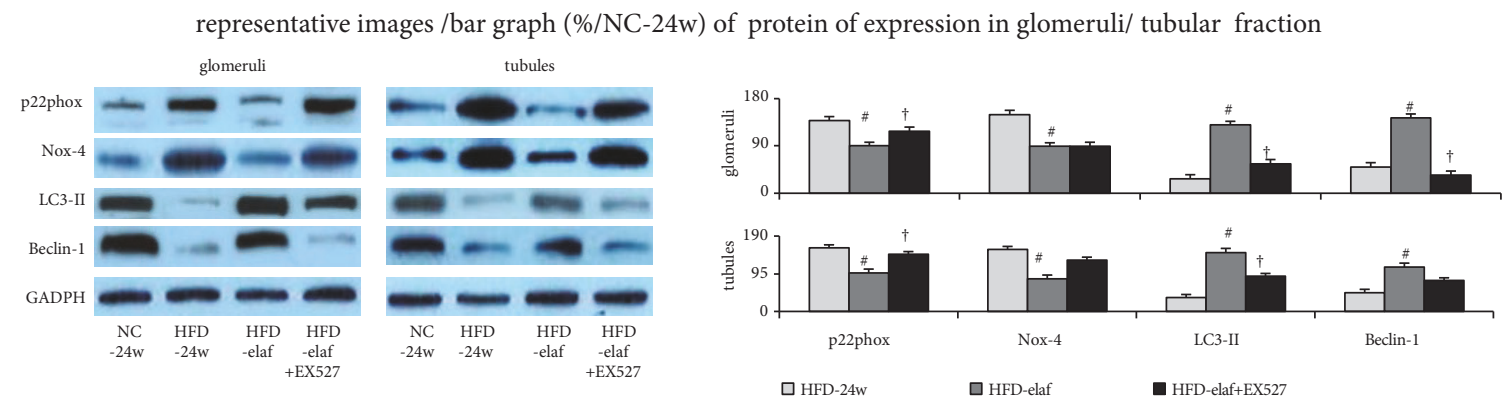

(e)

FIGURE 6: The suppression of renal oxidative stress was accompanied by the correction of autophagy impairment in HFD-induced CKD mice receiving chronic elafibranor treatment. For the quantitative evaluation, eight stained tissue sections slides and ten randomly taken pictures were included from each studied group. Representative IF images (200x)/bar graphs of glomerular synaptopodin (a) and renal tubules cubulin (b) expression. mRNAs expression in glomeruli (c) and renal tubules (d) fractions; (e) representative images/bar graphs of western blot for renal proteins expressions in glomeruli and tubular fractions. $* p<0.05$ versus NC-group; ${ }^{*} \mathrm{p}<0.05$ versus HFD-group; ${ }^{\dagger} \mathrm{p}<0.05$ versus $\mathrm{HFD}$ elaf group.

with CKD [27]. In our study, the effects and mechanisms of renoprotective effects of 12 weeks of elafibranor treatment were explored in HFD-fed NASH mice with CKD. Nonetheless, it is mandatory to explore the shortest period of renoprotective effects of elafibranor which need to be evaluated in future studies.

Antiapoptosis and antioxidative stress effects of SIRT1 are accompanied by its anti-inflammatory effects in animals with CKD and steatohepatitis [3, 5, 7, 14-19, 21, 28]. Hepatic and renal SIRT1 were reduced in animals with steatohepatitis and CKD $[14,15]$. In our study, concomitant SIRT1 inhibitor (EX527) treatment reversed elafibranor-related benefits, indicating that these effects are SIRT1-dependent. Primarily, antioxidative stress effect is responsible for the activation of renal SIRT1 in HFD-fed mice treated with elafibranor.

By increasing peroxisome function, SIRT1 activation, which is reciprocally stimulated by upregulated PPAR $\alpha$ and $\operatorname{PPAR} \delta$, can prevent the drug-induced renal cell apoptosis and acute kidney injury in mice [29-33]. Decreased SIRT1 expression on podocyte increases cell apoptosis and albuminuria in mice $[16,32]$. In our study, siSIRT1 coincubation eliminates PPAR $\alpha / \delta$ agonist elafibranor-related suppression of HFD-sera-induced apoptosis. Meanwhile, rSIRT1 coincubation has similar effects as elafibranor on the reversal of HFD-sera-induced apoptosis. So, in current study, the renoprotective effect of the PPAR $\alpha / \delta$ agonist elafibranor, at least partly, is attributable to SIRT1-mediated inhibition of HFD-induced circulating factors on renal cells and the kidney.

Autophagy promotes cell survival by elimination of damage organelles, which is initiated by increased Beclin-1/LC3II levels, resulting in increased autophagic flux. Suppression of autophagy (reduced Beclin-1/LC3-II) and autophagy flux induce cell apoptosis. PPAR $\alpha$ activation protects the liver from acute inflammation and failure by activating autophagy [33]. PPAR $\delta$ activation protects human cardiac cells from ER stress-induced injury by stimulating autophagy [34]. Inhibition SIRT1 exacerbates oxidative stress-suppressed autophagy 


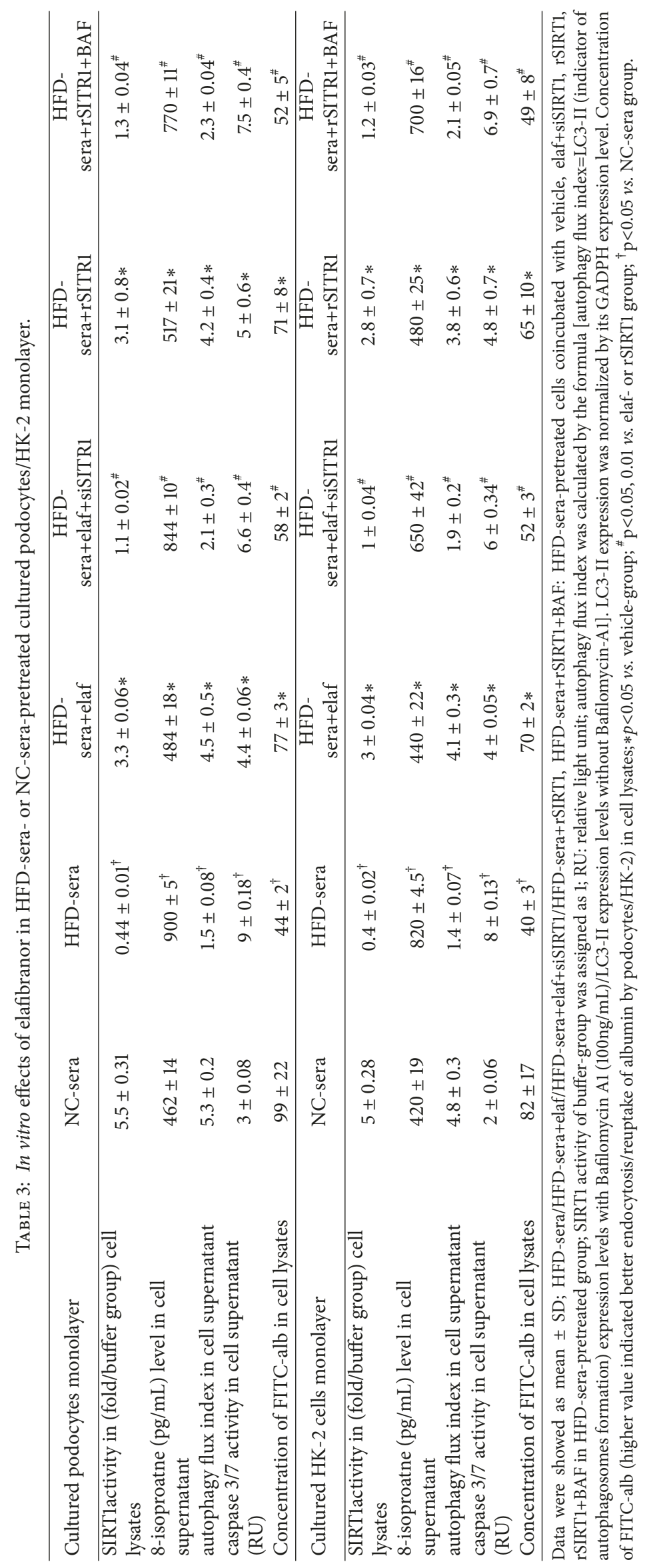




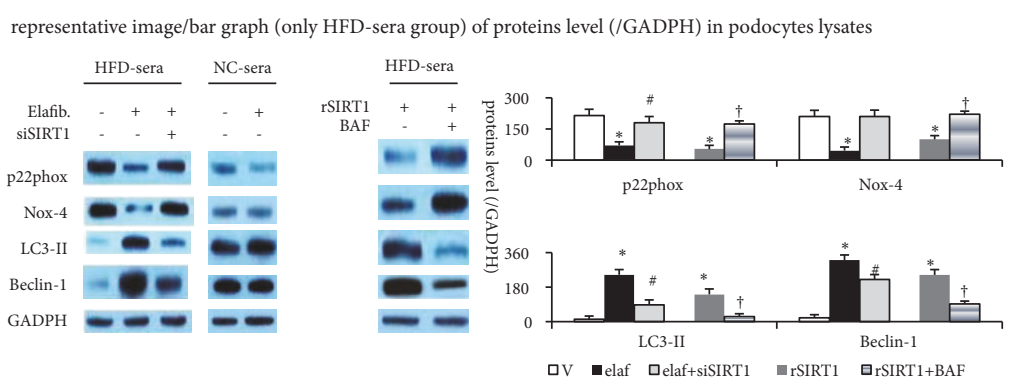

(a)

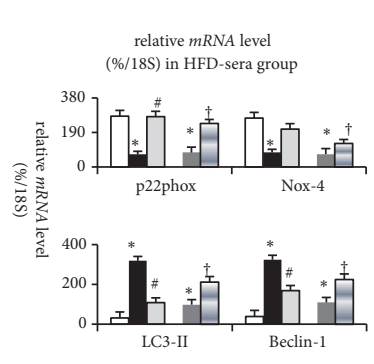

(b)

representative image/bar graph apoptotic podocytes (\%) in different treatments
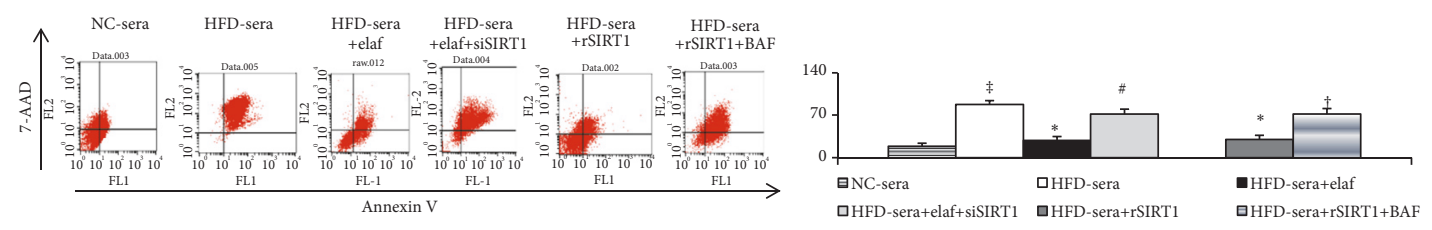

(c)
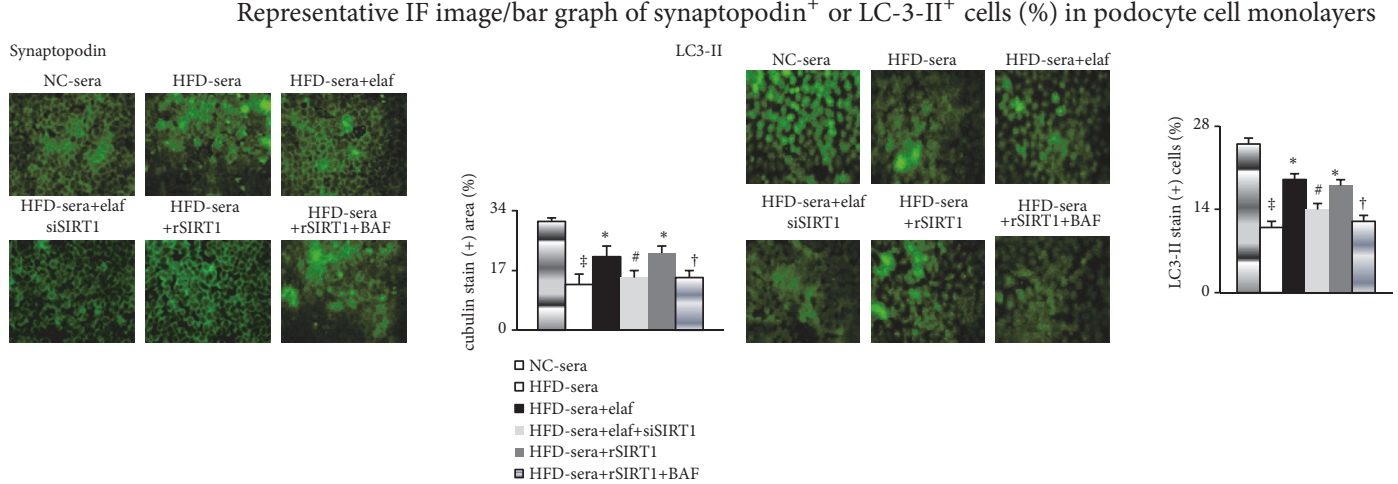

(d)

FIGURE 7: SIRT1-activated and autophagy-mediated effects of elafibranor on 10\% HFD-sera-pretreated oxidative stress and apoptosis in podocytes. (a) Proteins/(b) $m R N A$ levels in lysates of podocytes; (c) flow cytometry-assessed apoptotic cells; (d) IF image/bar graph of synaptopodin/LC3II expression in podocytes monolayer cells. The optical section data for $\%$ of synaptopodin/cubulin/LC3-II $(+)$ area on each slide were evaluated. $\neq \mathrm{p}<0.05$ versus $\mathrm{NC}$-sera group; $* p<0.05$ versus $\mathrm{V}$-group; ${ }^{\#} \mathrm{p}<0.05$ versus elaf-group; ${ }^{\dagger} \mathrm{p}<0.05$ versus rSIRT1-group.

in stem cells [35]. Downregulation of SIRT1 signals is involved in the HFD-induced renal dysfunction in mice [36]. SIRT1 activator suppresses hyperglycemia-induced apoptosis of podocytes via autophagy activation in diabetic mice with nephropathy [37]. In in vitro experiments, we revealed that the coincubation with autophagy flux blocker (BAF) abolished elafibranor-related SIRT1-mediated inhibition of NASH-sera-induced pathogenic signals in podocytes and HK-2 cells.

Impaired glomerular protein endocytosis and reduced tubular reuptake of leakage protein can lead to albuminuria. Podocytes are epithelial cells of the outer membrane of renal glomeruli that maintain its integrity. Podocytes damage not only impairs glomerular barrier but also collapses its architecture and leads to advanced renal injury and albuminuria. Restoration of glomerular barrier protein expressions including ZO-1 and synaptopodin (marker of podocyte) avoids albuminuria in diabetic mice [38]. Proximal tubule cells have a capacity to uptake glomeruli-leaked albumin and prevent final leakage. In proximal renal tubules, cubulin mediates the reuptake of leakage albumin from glomeruli to avoid tubulointerstitial inflammation/fibrosis [39]. Oxidative stress-related downregulation of barrier markers worsens albuminuria and tubulointerstitial inflammation/fibrosis [40, 41]. Our study revealed that CKD-related oxidative stress and albuminuria were associated with the downregulation of renal barrier markers in HFD-induced obese mice with steatohepatitis.

In summary, through systemic in vivo and in vitro approaches, our study revealed that kidney-specific protective effects of elafibranor are attributable to the preservation of glomerular/tubular barrier protein, maintenance of structure, antioxidative stress, and antiapoptosis effects via activation of SIRT-autophagy-mediated protective signals (Figure 9).

This study suggested that elafibranor and strategies aimed at activating SIRT-autophagy are promise for treating high fat consumption which induces steatohepatitis and CKD. 
representative image/bar graph (only HFD-sera group) of proteins level /GADPH) in HK-2 cell lysates
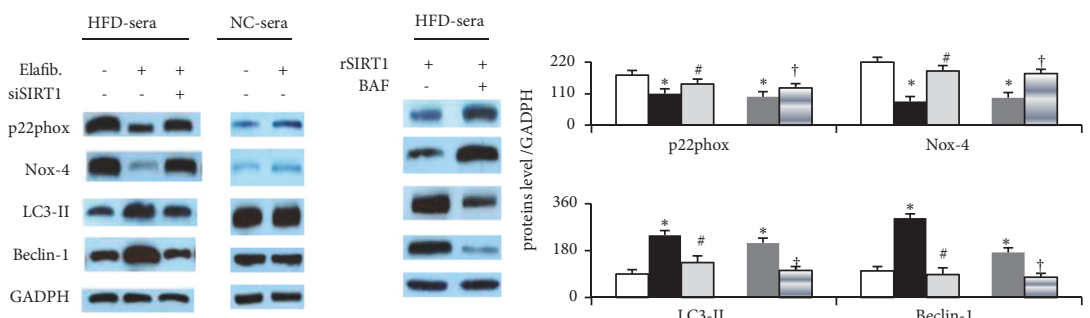

relative $m R N A$ level $(\% / 18 \mathrm{~S})$ in HFD-sera group

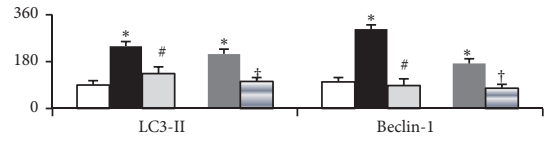

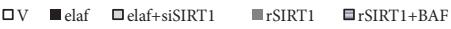

(a)

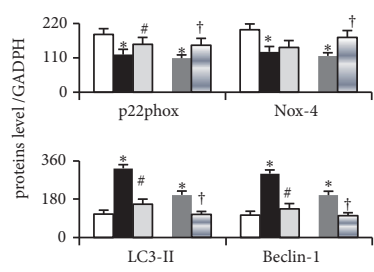

(b)

representative image/bar graph apoptotic HK-2 cells (\%) in different treatments
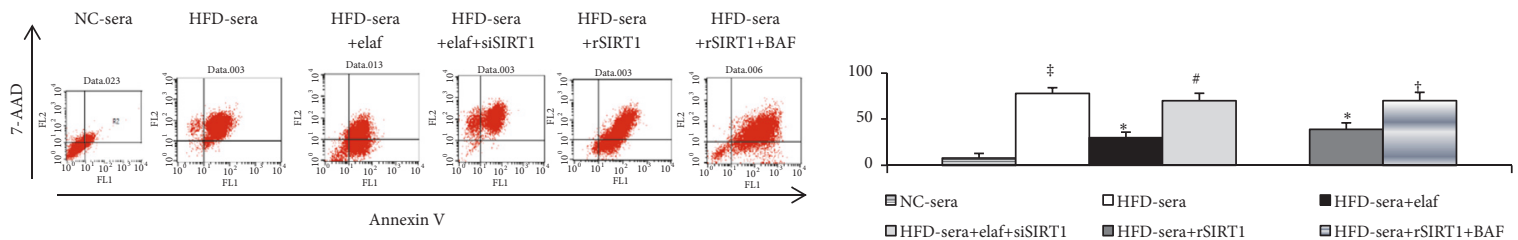

(c)

Representative IF image/bar graph of cubulin ${ }^{+} / \mathrm{LC}^{-3}-\mathrm{II}^{+}$cells (\%) in HK-2 cell monolayers

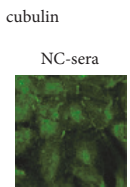

HFD-sera+ela

HFD-sera+el
siSIRT1

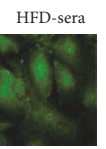

HFD-sera

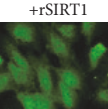

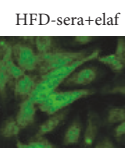

HFD-sera

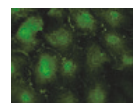

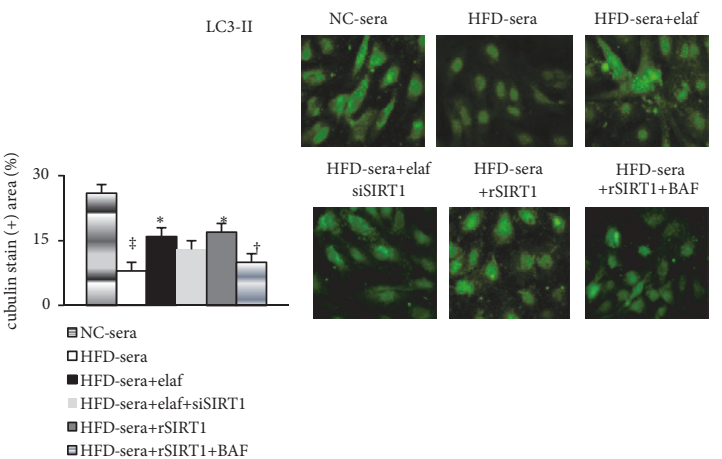

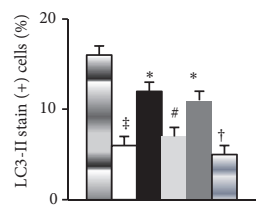

(d)

FIGURE 8: SIRT1-activated and autophagy-mediated effects of elafibranor on 10\% HFD-sera-increased oxidative stress and apoptosis in HK-2 cells. (a) Proteins/(b) mRNA levels in HK-2 cell lysates; (c) flow cytometry-assessed apoptotic cells; (d) IF image/bar graph of cubulin/LC3II expression in HK-2 monolayer cells. $\ddagger \mathrm{p}<0.05$ versus NC-sera group; $* p<0.05$ versus V-group; ${ }^{\#} \mathrm{p}<0.05$ versus elaf-group; ${ }^{\dagger} \mathrm{p}<0.05$ versus rSIRT1 group.

\section{Data Availability}

All data supporting the results reported in the article can be found in Division of General Medicine, Department of Medicine, Taipei Veterans General Hospital, Taipei, Taiwan, and can be seen after asking the corresponding author.

\section{Conflicts of Interest}

The authors declare that there are no conflicts of interest regarding the publication of this article.

\section{Authors' Contributions}

Ying-Ying Yang and Han-Chieh Lin contributed equally to this work.

\section{Acknowledgments}

We especially thank Yun-Ru Wang, Fan-Yi Jhan, Huei-Ting Yang, Che-Jui Chang, and Yen-Ling Lin for their excellent technical support. This work was supported by grants NSC-102-2314-B-010-036-MY3 and MOST-2314-B-010-024MY3 from the National Science Council, and V106C-007, VGHUST105-G1-2-2, and by the Taipei Veterans General Hospital.

\section{Supplementary Materials}

Among different groups of male C57BL/6 mice, the whole study includes measurements of serum and tissue metabolic and inflammatory profiles, microscopic analysis of renal tissue for severity of NASH, apoptosis, autophagy and cubulin/synaptopodin, protein and mRNA expressions of 


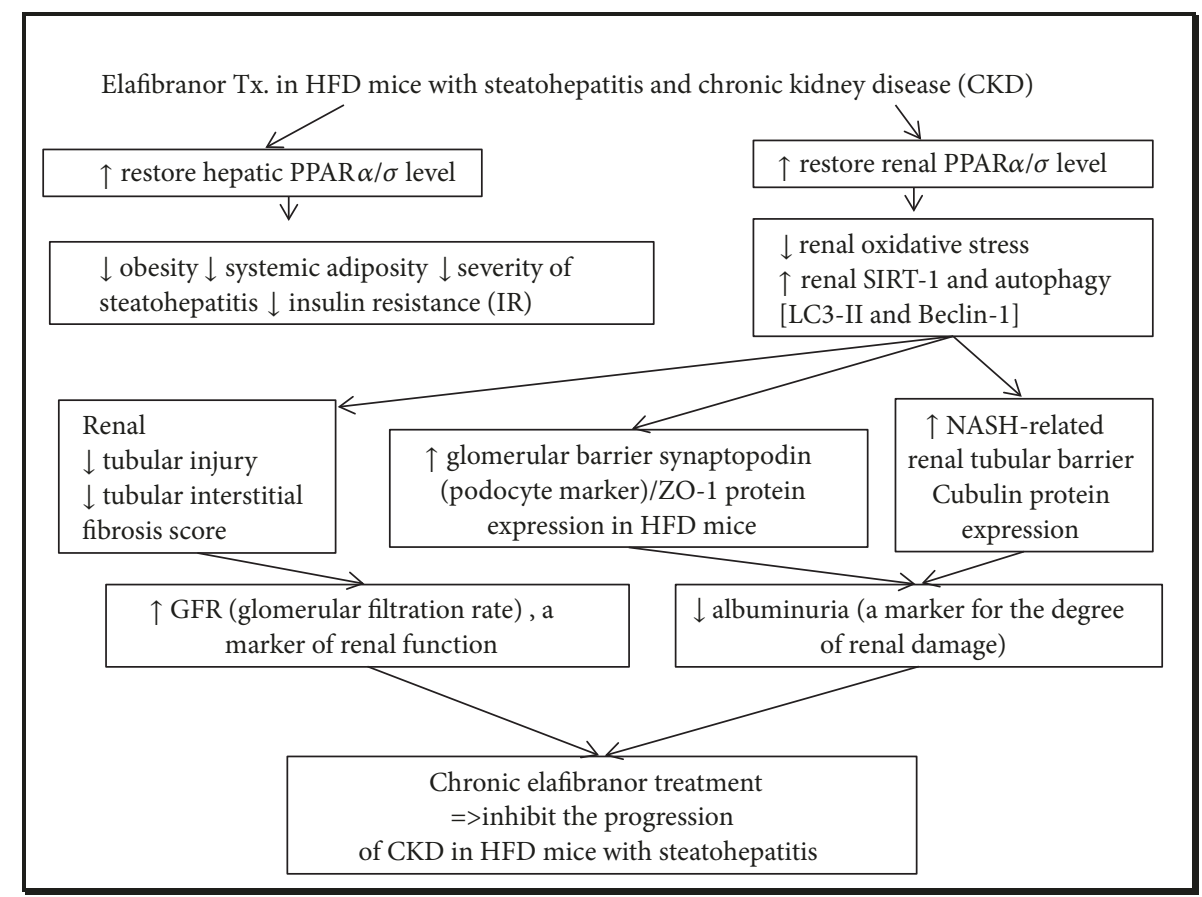

FIGURE 9: Schematic representative hypothesis for the mechanisms of the inhibition of the progressive of chronic kidney disease (CKD) by chronic elafibranor treatment on HFD mice with steatohepatitis in our study.

various barrier and autophagic markers. Additionally, the roles of SIRT1-autophagy on elafibranor-related effects were evaluated in HFD-sera-pretreated podocytes and HK-2 cells system. The additional detail materials and methods are included in supplementary materials and methods. (Supplementary Materials)

\section{References}

[1] M. V. Machado, S. Gonçalves, F. Carepa, J. Coutinho, A. Costa, and H. Cortez-Pinto, "Impaired renal function in morbid obese patients with nonalcoholic fatty liver disease," Liver International, vol. 32, no. 2, pp. 241-248, 2012.

[2] J. Lin, S. Judd, A. Le et al., "Associations of dietary fat with albuminuria and kidney dysfunction," American Journal of Clinical Nutrition, vol. 92, no. 4, pp. 897-904, 2010.

[3] E. Börgeson and K. Sharma, "Obesity, immunomodulation and chronic kidney disease," Current Opinion in Pharmacology, vol. 13, no. 4, pp. 618-624, 2013.

[4] A. K. Singal, M. Hasanin, M. Kaif, R. Wiesner, and Y.-F. Kuo, "Nonalcoholic steatohepatitis is the most rapidly growing indication for simultaneous liver kidney transplantation in the United States," Transplantation, vol. 100, no. 3, pp. 607-612, 2016.

[5] P. Stenvinkel, C. Zoccali, and T. A. Ikizler, "Obesity in CKD—what should nephrologists know?" Journal of the American Society of Nephrology, vol. 24, no. 11, pp. 1727-1736, 2013.

[6] P. Kincaid-Smith, "Hypothesis: obesity and the insulin resistance syndrome play a major role in end-stage renal failure attributed to hypertension and labelled 'hypertensive nephrosclerosis," Journal of Hypertension, vol. 22, no. 6, pp. 1051-1055, 2004.
[7] N. Deji, S. Kume, S.-I. Araki et al., "Structural and functional changes in the kidneys of high-fat diet-induced obese mice," American Journal of Physiology-Renal Physiology, vol. 296, no. 1, pp. F118-F126, 2009.

[8] M. Pawlak, P. Lefebvre, and B. Staels, "Molecular mechanism of PPAR $\alpha$ action and its impact on lipid metabolism, inflammation and fibrosis in non-alcoholic fatty liver disease," Journal of Hepatology, vol. 62, no. 3, pp. 720-733, 2015.

[9] U. Risérus, D. Sprecher, T. Johnson et al., "Activation of peroxisome proliferator-activated receptor (PPAR)delta promotes reversal of multiple metabolic abnormalities, reduces oxidative stress, and increases fatty acid oxidation in moderately obese men," Diabetes, vol. 57, no. 2, pp. 332-339, 2008.

[10] B. Staels, A. Rubenstrunk, B. Noel et al., "Hepatoprotective effects of the dual peroxisome proliferator-activated receptor alpha/delta agonist, GFT505, in rodent models of nonalcoholic fatty liver disease/nonalcoholic steatohepatitis," Hepatology, vol. 58, no. 6, pp. 1941-1952, 2013.

[11] C. W. Park, Y. Zhang, X. Zhang et al., "PPAR $\alpha$ agonist fenofibrate improves diabetic nephropathy in db/db mic," Kidney International, vol. 69, no. 9, pp. 1511-1517, 2006.

[12] V. Ratziu, S. A. Harrison, S. Francque et al., "Elafibranor, an agonist of the peroxisome proliferator-activated receptor-a and $-\mathrm{d}$, induces resolution of nonalcoholic steatohepatitis without fibrosis worsening," Gastroenterology, vol. 150, no. 5, pp. 11471159, 2016.

[13] E. Y. Lee, G. T. Kim, M. Hyun et al., "Peroxisome proliferatoractivated receptor- $\delta$ activation ameliorates albuminuria by preventing nephrin loss and restoring podocyte integrity in type 2 diabetes," Nephrology Dialysis Transplantation, vol. 27, no. 11, pp. 4069-4079, 2012.

[14] P. T. Pfluger, D. Herranz, S. Velasco-Miguel, M. Serrano, and M. H. Tschöp, "Sirtl protects against high-fat diet-induced 
metabolic damage," Proceedings of the National Acadamy of Sciences of the United States of America, vol. 105, no. 28, pp. 9793-9798, 2008.

[15] X.-Q. Deng, L.-L. Chen, and N.-X. Li, “The expression of SIRT1 in non-alcoholic fatty liver disease induced by high-fat diet in rats," Liver International, vol. 27, no. 5, pp. 708-715, 2007.

[16] K. Hasegawa, S. Wakino, and P. Simic, "Renal tubular SIRT1 attenuates diabetic albuminuria by epigenetically suppressing claudin-1 over-expression in podocytes," Nature Medicine, vol. 19, no. 11, pp. 1496-1504, 2013.

[17] X. Z. Huang, D. Wen, M. Zhang et al., "Sirtl activation ameliorates renal fibrosis by inhibiting the TGF- $\beta / S \operatorname{mad} 3$ pathway," Journal of Cellular Biochemistry, vol. 115, no. 5, pp. 996-1005, 2014.

[18] J. M. O. Andrade, A. F. Paraíso, M. V. M. de Oliveira et al., "Resveratrol attenuates hepatic steatosis in high-fat fed mice by decreasing lipogenesis and inflammation," Nutrition Journal, vol. 30, no. 7-8, pp. 915-919, 2014.

[19] H. Moridi, J. Karimi, N. Sheikh et al., "Resveratrol-dependent down-regulation of receptor for advanced glycation endproducts and oxidative stress in kidney of rats with diabetes," International Journal of Endocrinology and Metabolism, vol. 13, no. 2, Article ID e23542, 2015.

[20] D. Kim, J. E. Lee, Y. J. Jung et al., "Metformin decreases highfat diet-induced renal injury by regulating the expression of adipokines and the renal AMP-activated protein kinase/acetylCoA carboxylase pathway in mice," International Journal of Molecular Medicine, vol. 32, no. 6, pp. 1293-1302, 2013.

[21] N. Deji, S. Kume, S.-I. Araki et al., "Structural and function changes in the kidney of high-fat diet-induced obese mice," American Journal of Physiology-Renal Physiology, vol. 296, no. 1, pp. F118-F126, 2009.

[22] A. S. Levey, C. Becker, and L. A. Inker, "Glomerular filtration rate and albuminuria for detection and staging of acute and chronic kidney disease in adults: a systematic review," The Journal of the American Medical Association, vol. 313, no. 8, pp. 837-846, 2015.

[23] C. W. Park, H. W. Kim, S. H. Ko et al., "Accelerated diabetic nephropathy in mice lacking the peroxisome proliferatoractivated receptor," Diabetes, vol. 55, no. 4, pp. 885-893, 2006.

[24] H. H. Chen, Y. M. Sue, C. H. Chen et al., "Peroxisome proliferator-activated receptor alpha plays a crucial role in Lcarnitine anti-apoptosis effect in renal tubular cells," Nephrology, Dialysis, Transplantation, vol. 24, no. 10, pp. 3042-3049, 2009.

[25] X. Yang, S. Kume, Y. Tanaka et al., “GW501516, a PPAR $\delta$ agonist, ameliorates tubulointerstitial inflammation in proteinuric kidney disease via inhibition of TAK1-NF $\kappa$ B pathway in mice," PLoS ONE, vol. 6, no. 9, Article ID e25271, 2011.

[26] M. Pesant, S. Sueur, P. Dutartre et al., "Peroxisome proliferatoractivated receptor $\delta(\operatorname{PPAR} \delta)$ activation protects $\mathrm{H} 9 \mathrm{c} 2$ cardiomyoblasts from oxidative stress-induced apoptosis," Cardiovascular Research, vol. 69, no. 2, pp. 440-449, 2006.

[27] Y. Tanaka, S. Kume, S. Araki et al., "Fenofibrate, a PPAR $\alpha$ agonist, has renoprotective effects in mice by enhancing renal lipolysis," Kidney International, vol. 79, no. 8, pp. 871-882, 2011.

[28] K. Hasegawa, S. Wakino, K. Yoshioka et al., "Kidney-specific overexpression of Sirtl protects against acute kidney injury by retaining peroxisome function," The Journal of Biological Chemistry, vol. 285, no. 17, pp. 13045-13056, 2010.
[29] M. Okazaki, Y. Iwasaki, M. Nishiyama et al., "PPAR $\beta / \delta$ regulates the human SIRT1 gene transcription via Sp1," Endocrine Journal, vol. 57, no. 5, pp. 403-413, 2010.

[30] S. Hayashida, A. Arimoto, Y. Kuramoto et al., "Fasting promotes the expression of SIRT1, an NAD(+)-dependent protein deacetylase, via activation of PPARalpha in mice," Molecular and Cellular Biochemistry, vol. 339, no. 1-2, pp. 285-292, 2010.

[31] P. Y. Chuang, W. Cai, X. Li et al., "Reduction in podocyte SIRT1 accelerates kidney injury in aging mice," American Journal of Physiology-Renal Physiology, vol. 313, no. 3, pp. F621-F628, 2017.

[32] G. Miglio, A. C. Rosa, L. Rattazzi et al., “The subtypes of peroxisome proliferator-activated receptors expressed by human podocytes and their role in decreasing podocyte injury," British Journal of Pharmacology, vol. 162, no. 1, pp. 111-125, 2011.

[33] M. Jiao, F. Ren, L. Zhou et al., "Peroxisome proliferator-activated receptor $\alpha$ activation attenuates the inflammatory response to protect the liver from acute failure by promoting the autophagy pathway," Cell Death \& Disease, vol. 5, Article ID e1397, 2014.

[34] X. Palomer, E. Capdevila-Busquets, G. Botteri et al., "PPAR $\beta / \delta$ attenuates palmitate-induced endoplasmic reticulum stress and induces autophagic markers in human cardiac cells," International Journal of Cardiology, vol. 174, no. 1, pp. 110-118, 2014.

[35] X. Ou, M. R. Lee, X. Huang, S. Messina-Graham, and H. E. Broxmeyer, "SIRT1 positively regulates autophagy and mitochondria function in embryonic stem cells under oxidative stress," Stem Cells, vol. 32, no. 5, pp. 1183-1194, 2014.

[36] N. Zhang, Z. Li, K. Xu, Y. Wang, and Z. Wang, "Resveratrol protects against high-fat diet induced renal pathological damage and cell senescence by activating SIRT1," Biological \& Pharmaceutical Bulletin, vol. 39, no. 9, pp. 1448-1454, 2016.

[37] S. Huang, D. Ding, S. Chen et al., "Resveratrol protects podocytes against apoptosis via stimulation of autophagy in a mouse model of diabetic nephropathy," Scientific Reports, vol. 7, no. 1, article 45692, 2017.

[38] H. Rincon-Choles, T. L. Vasylyeva, P. E. Pergola et al., "ZO1 expression and phosphorylation in diabetic nephropathy," Diabetes, vol. 55, no. 4, pp. 894-900, 2006.

[39] S. Amsellem, J. Gburek, G. Hamard et al., "Cubilin is essential for albumin reabsorption in the renal proximal tubule," Journal of the American Society of Nephrology, vol. 21, no. 11, pp. 1859$1867,2010$.

[40] K. Sharma, S. RamachandraRao, G. Qiu et al., "Adiponectin regulates albuminuria and podocyte function in mice," The Journal of Clinical Investigation, vol. 118, no. 5, pp. 1645-1656, 2008.

[41] H. Birn, J. C. Fyfe, C. Jacobsen et al., "Cubilin is an albumin binding protein important for renal tubular albumin reabsorption," The Journal of Clinical Investigation, vol. 105, no. 10, pp. 1353-1361, 2000. 


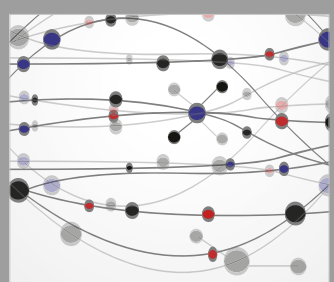

The Scientific World Journal
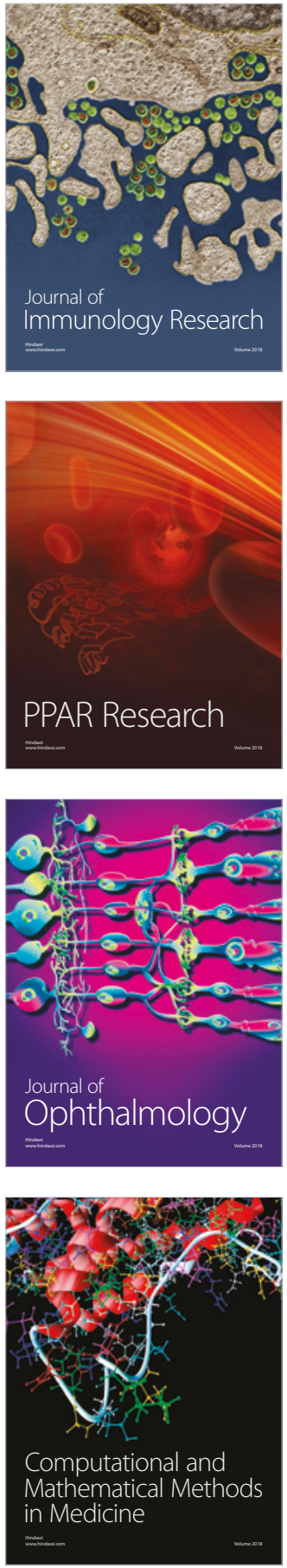

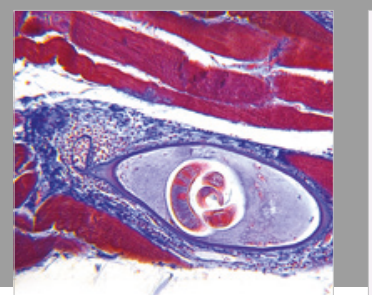

Gastroenterology Research and Practice

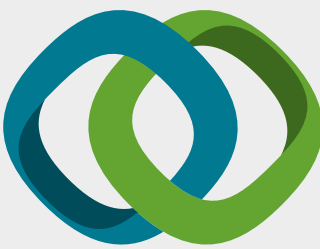

\section{Hindawi}

Submit your manuscripts at

www.hindawi.com
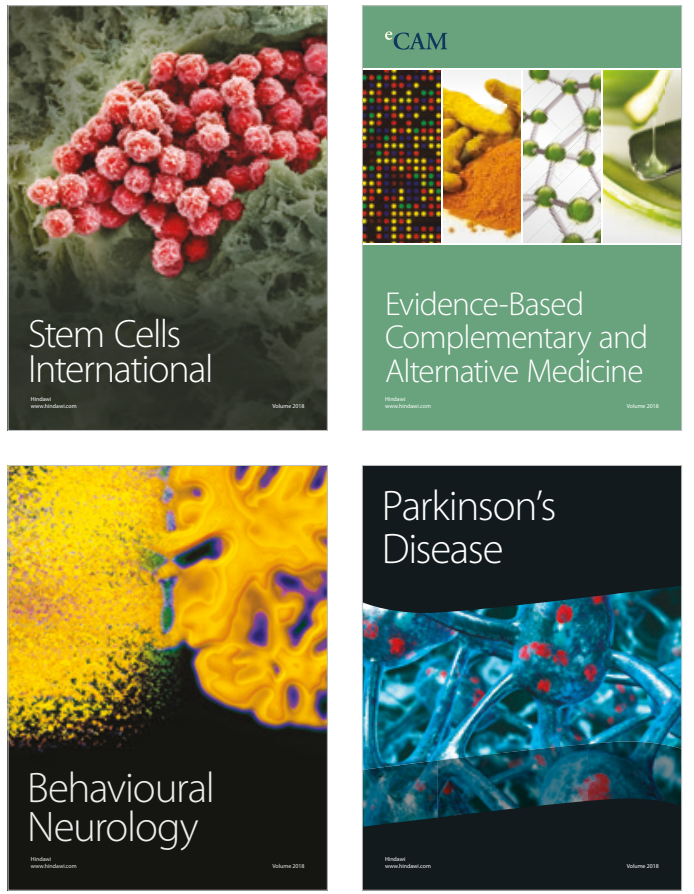

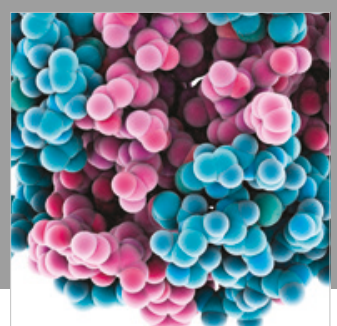

ournal of

Diabetes Research

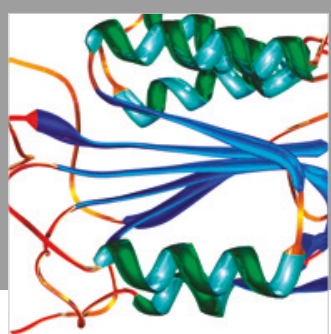

Disease Markers
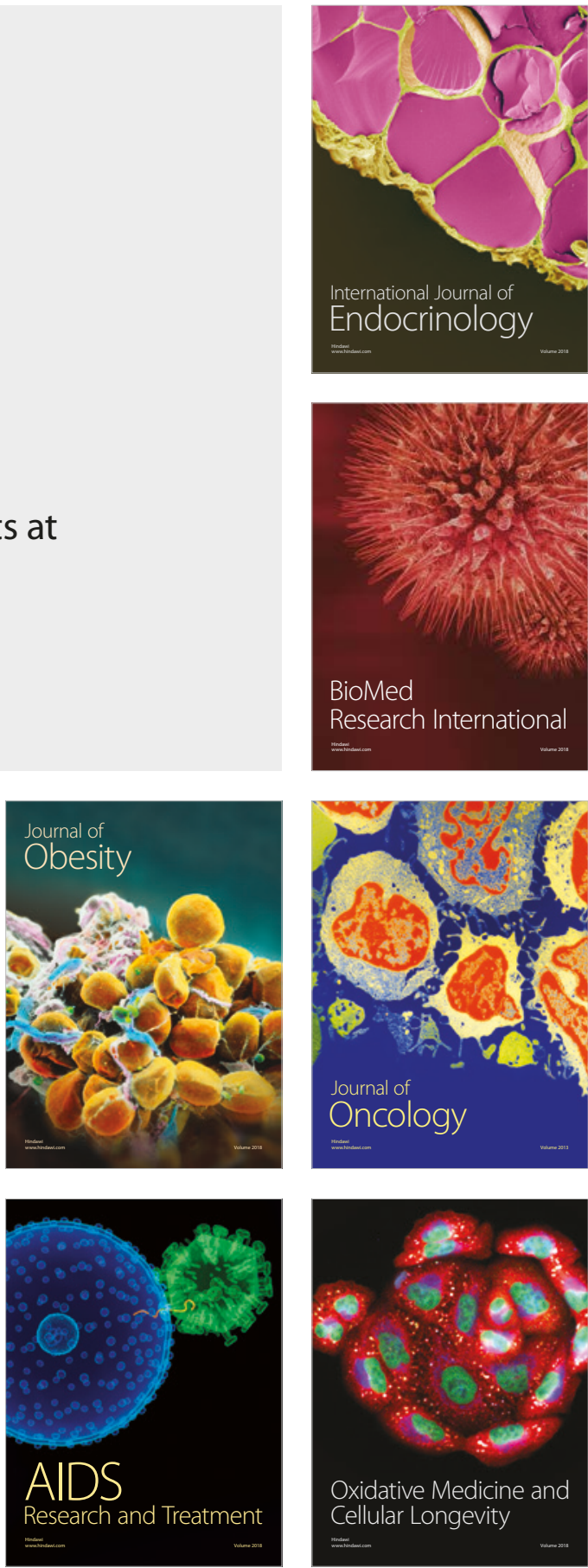